\title{
Dimensionless Size Scaling of Intrinsic Rotation in DIII-D
}

\author{
J.S. deGrassie ${ }^{1}$, W.M. Solomon ${ }^{1}$, J.E. Rice ${ }^{2}$, and J.-M. Noterdaeme ${ }^{3,4}$ \\ ${ }^{1}$ General Atomics, P.O. Box 85608, San Diego, California 92186-5608, USA \\ ${ }^{2}$ Plasma Science and Fusion Center, MIT, Cambridge, Massachusetts 02139, USA \\ ${ }^{3}$ Max-Planck Institute for Plasma Physics, EURATOM Association, Boltzmannstr. 2, 85748 Garching, \\ Germany \\ ${ }^{4}$ University of Gent, Belgium
}

\begin{abstract}
A dimensionless empirical scaling for intrinsic toroidal rotation is given; $\mathrm{M}_{\mathrm{A}} \sim \beta_{\mathrm{N}} \rho^{*}$, where $M_{A}$ is the toroidal velocity divided by the Alfvén velocity, $\beta_{N}$ the usual normalized $\beta$ value, and $\rho^{*}$ is the ion gyroradius divided by the minor radius. This scaling describes well experimental data from DIII-D, and also some published data from C-Mod and JET. The velocity used in this scaling is in an outer location in minor radius, outside of the interior core and inside of the large gradient edge region in H-mode conditions. This scaling establishes the basic magnitude of the intrinsic toroidal rotation and its relation to the rich variety of rotation profiles that can be realized for intrinsic conditions is discussed. This scaling has some similarities to existing dimensioned scalings, both the Rice scaling [J.E. Rice et al, Phys. Plasmas 7, 1825 (2000)] and the scaling of Parra et al [F.I. Parra et al, Phys. Rev. Lett. 108, 095001 (2012)]. These relationships are described.
\end{abstract}

\section{Introduction}

A present goal within magnetic fusion energy research is verification and validation of powerful computational models based upon physical models of the complex processes at work in high temperature confined plasmas [1]. Such codes are looked to for predictive capability, for example in designing experimental scenarios and eventually new devices. Yet, the validation process by nature involves "imperfect" models and solutions [1], whereas the experiments work with "perfect" models, i.e. reality, although the measurements are neither perfect, nor complete [1]. The tokamak path toward energy production has successfully developed upon the ability to find predictive capabilities 
through the experiments themselves, that is, empirical scalings, notably for global energy confinement and for global stability limits. The empirical energy confinement time scaling law "is the basis on which new tokamaks are designed" [2]. Empirical scaling can be cast in either the so-called machine parameters, dimensioned quantities, or in the dimensionless parameters that underlie the governing physical equations applicable for the experiments [3], the latter likely providing greater insight regarding the dominant underlying physical processes.

Here, we take a step in establishing a scaling for tokamak intrinsic rotation. This is the toroidal rotation observed to exist in an axisymmetric device with no injected toroidal momentum source. Unlike the plasma particle density and energy that are scalar quantities the canonical toroidal angular momentum is a vector quantity, greatly enhancing the diversity of profiles that are theoretically possible, and indeed are measured experimentally [4-6]. Single scalar quantities such as average density or total stored energy would seemingly have less value in defining momentum. The rotation profile is important. It is recognized that not only the absolute local magnitude of rotation but also the radial shear of rotation are both important in relation to stability and confinement $[7,8]$.

Nevertheless, we present a single parameter, local, dimensionless scaling for intrinsic rotation in DIII-D. Published data from JET [9] and C-Mod [4] also fit this scaling. In machine parameters, this scaling is related to the ion temperature dominant scaling postulated by Parra et al on physical grounds [10], where $T_{i}$ is the ion temperature. We show rotation profiles to indicate how our scaling value appears across the minor radius.

Commonly speaking we refer to toroidal rotation, $\omega_{\phi}$, whereas we measure the toroidal flow velocity of an ion species, $V_{\phi}=R \omega_{\phi}$, where $R$ is the major radius. We will use both terms somewhat interchangeably. 


\section{Velocity Profile Considerations}

The variety in intrinsic rotation profiles has become better defined since the early experiments on intrinsic rotation. A typical ELMing H-mode generally has intrinsic rotation in the direction of the plasma current, co- $\mathrm{I}_{\mathrm{p}}$, which we define as positive in sign. But there can be a depression of rotation, or actual reversals, in the core that are in DIII-D associated with electron heating $(\mathrm{ECH})$ [4]. Dramatic core reversals in L-mode that depend on density and $\mathrm{I}_{\mathrm{p}}$ magnitude have been investigated in TCV [5] and C-Mod [6]. A review of possible theoretical causes of reversals and general intrinsic rotation considerations has been given by Diamond et al [11]. Some effects that could lead to reversals are changes in the dominant turbulence mechanism and/or collisionality [12], or by a change in collisionality coupled with turbulence [13], or turbulence and q-shear [14].

Additionally it has become established that a large, co- $\mathrm{I}_{\mathrm{p}}$, narrow bulk ion velocity layer can exist at the outboard edge in the tokamak [15-17]. This effect has been explained as due to thermal ion orbit loss $[18,19]$ or neoclassical orbit effects coupled with orbit loss and turbulence [20]. The relation of this edge flow layer to global intrinsic rotation is still being investigated experimentally [17].

Armed with some understanding of the phenomena leading to non-monotonic rotation profiles in DIII-D allows us to seek a scaling based upon only a single minor radius value of rotation, at a radial location where the rotation is ideally least subject to these other phenomena that drive momentum rearrangement within the profile. The location in minor radius need not be specified to high precision as will be shown in the data. 


\section{Background: Empirical and Dimensionless Scaling of Intrinsic Rotation}

At C-Mod, Rice and colleagues did some of the first experiments on intrinsic rotation in diverted tokamaks [21-23]. These experimental measurements were made in the interior in minor radius and an on-axis toroidal velocity was usually reported. The measurements of $\mathrm{V}_{\phi}$ were made using $\mathrm{X}$-ray spectra from trace injection of argon, and their experiments over time have shown this to be a good indicator of the bulk ion core velocity in C-Mod [24]. A machine parameter scaling for $V_{\phi}$ was found where the magnitude of the positive core toroidal velocity increased linearly with $\mathrm{W} / \mathrm{I}_{\mathrm{p}}$, where $\mathrm{W}$ is the total plasma stored energy and $I_{p}$ is the magnitude of the plasma current [21-23]. This is referred as "Rice scaling" for intrinsic rotation.

Subsequently, intrinsic rotation experiments were performed in DIII-D, with ECH Hmodes, L-modes, Ohmic H-modes and simply Ohmic discharges [4,25,26]. In DIII-D short neutral beam injection (NBI) blips must be used for Charge Exchange Recombination (CER) Spectroscopy [27] velocity (and ion temperature) measurements of the minority impurity $\mathrm{C}^{6+}$ ion. In the original experiments all DIII-D beams pointed in one toroidal direction. In 2006 two NB lines were reversed, allowing the subsequent development of balanced NBI blips for intrinsic rotation CER measurements. Recently a main ion (e.g. $\mathrm{D}^{+}$) CER capability has been developed in DIII-D [28]. Measurements have shown that while in the edge, $\rho>0.85$, there can be a significant difference in the toroidal velocities $\mathrm{D}^{+}$and $\mathrm{C}^{6+}$, inside of this radius the two velocities are reasonably matched [28]. The bulk ion edge flow layer and the stronger pressure gradients in the edge are the likely causes of the edge differences in $V_{\phi}$ for the two species.

The DIII-D discharges with ECH were observed to show the co- $\mathrm{I}_{\mathrm{p}} \mathrm{V}_{\phi}$ in the outer $1 / 3$ or so of minor radius but had varying degrees of depression, or actual reversal of $V_{\phi}$ in the core. The closer to the magnetic axis the ECH deposition location, the more the tendency for core reversal [4], indicating that direct electron heating could be the cause [11, 12,29]. Coming from the other direction, from the edge boundary into the core plasma, the 
positive edge flow layer is observed typically to decrease going inward and then turns upward, more positive [15-17]. With a depressed core rotation this results in a peak in the co- $\mathrm{I}_{\mathrm{p}} \mathrm{V}_{\phi}$, in the general vicinity of $\rho \sim 0.75$. [4]. It was found that the velocity in this region followed a Rice scaling in DIII-D [4], albeit with a different proportionality constant, i.e. slope, than in C-Mod. In DIII-D the directly measured values of $\mathrm{V}_{\phi}$ and $\mathrm{W}$ are used rather than a difference taken from a lower $\mathrm{W}$ point as in C-mod. It may be that this region in minor radius is determined by where the strong inward edge momentum pinch velocity is decreasing significantly in magnitude [30].

Finding a Rice scaling in DIII-D was an important step in establishing that a universal underlying cause exists, in contrast to some effect related to the details of a specific tokamak, such as residual non-axisymmetries in the applied fields or in the physical boundaries. Notably, a DIII-D experiment with CER measurements of bulk ion intrinsic $\mathrm{V}_{\phi}$ in helium discharges gave values that followed the same DIII-D Rice scaling [4], indicating that it applies for the main ions.

The commonality of a $\mathrm{W} / \mathrm{I}_{\mathrm{p}}$ scaling for intrinsic velocity between C-Mod and DIII-D led to a joint dimensionless similarity experiment between the two devices [4]. Here, matched dimensionless parameter discharges showed agreement for the toroidal thermal Mach value, $\mathrm{M}_{\phi} \equiv \mathrm{V}_{\phi} / \overline{\mathrm{V}}$, where $\overline{\mathrm{V}}$ is the ion thermal velocity, $\overline{\mathrm{V}} \equiv \sqrt{\mathrm{T}_{\mathrm{i}} / \mathrm{M}_{\mathrm{i}}}$. In the comparison discharges the dimensionless kinetic parameters near the magnetic axis were matched since this is the location for these C-Mod $\mathrm{V}_{\phi}$ measurements. The methodology adopted for comparison is that the outer "peak" velocity in DIII-D would diffusively soak in to the axis were it not for the effect of $\mathrm{ECH}$ on hollowing the core velocity profile, so we compare the DIII-D peak $\mathrm{M}_{\phi}$ with the core value in C-Mod, as shown in figure 1, which is taken from figure 9 of [4]. Well matched are the $\mathrm{q}_{95}$ and $\hat{\beta}$ values, whereas for size, $\hat{\rho}$, and collisionality, $\hat{v}$, the C-MoD values were $\sim 30 \%$ larger than in DIII-D (See Table I of reference 4, for shots labeled 1 and 2 , and for the definitions of $\widehat{\beta}, \hat{\rho}$ and $\hat{v}$ ). In the higher density conditions of C-Mod, $\mathrm{T}_{\mathrm{i}} \approx \mathrm{T}_{\mathrm{e}}$, the electron temperature, so $\mathrm{T}_{\mathrm{e}}$ was used to compute these dimensionless quantities in DIII-D also, but in the DIII-D 
discharge $T_{\mathrm{e}}$ is a bit higher than $\mathrm{T}_{\mathrm{i}}$ in the core due to the ECH. In C-Mod, other discharge conditions were run to show that $\mathrm{M}_{\phi} \sim \beta \mathrm{q}_{95}$ with the value of $\mathrm{M}_{\phi}$ changing with the toroidal field, $\mathrm{B}_{\mathrm{T}}[4]$.

This dimensionless parameter experiment between DIII-D and C-MoD shows that a dimensionless scaling in some form likely exists, but was not definitive since the amount of parameter space accessed was limited. Rice et al. investigated a number of possible scalings using data from several tokamaks [31], motivated by the original W/Ip scaling. DIII-D data from [4] are included here. The database has significantly fewer entries than those used for energy confinement scaling [32] but nevertheless indicated some interesting correlations. It is pointed out that if one uses an "MKS" version of $\beta_{\mathrm{N}}$, that is, $\beta_{\mathrm{N}}^{\mathrm{M}} \equiv \beta \mathrm{aB} / \mu_{0} \mathrm{I}_{\mathrm{p}}$, the database shows the correlation $\mathrm{M}_{\mathrm{A}} \approx \beta_{\mathrm{N}}^{\mathrm{M}}$, with roughly unity proportionality. Here, $\mathrm{M}_{\mathrm{A}}$ is the Alfvén "Mach value", $\mathrm{M}_{\mathrm{A}} \equiv \mathrm{V}_{\phi} / \mathrm{V}_{\mathrm{A}}$, with $\mathrm{V}_{\mathrm{A}} \equiv \mathrm{B}_{0} / \sqrt{\mu_{0} \mathrm{M}_{\mathrm{i}} \mathrm{n}_{\mathrm{i}}}$, with $\mathrm{B}_{0}$ the magnetic field on axis and $\mathrm{n}_{\mathrm{i}}$ an averaged ion density. It was this observation that guided the evaluation of a dimensionless scaling for intrinsic rotation in DIII-D data described in Section IV.

Another experimental observation that guided this search for a dimensionless scaling is the correlation of the intrinsic toroidal velocity with the local $\mathrm{T}_{\mathrm{i}}[10,19]$ or the gradient in $T_{i}$ [33]. This correlation also holds in conditions with significant NBI-torque drive $[34,35]$.

A plot of measured $V_{\phi}$ versus $T_{i}$ for the entire DIII-D dataset that will be described in Section IV is shown in Figure 2. These data cover the entire radial profile for each of the 24 discharges that comprise the set and each measurement time within a discharge, over 38,000 datapoints in all. There are times with only Ohmic, or ECH auxiliary heating that are strictly intrinsic, and there are times with steady beams at a low net toroidal torque, from -0.7 to $+0.9 \mathrm{Nm}$. The correlation of the local $\mathrm{V}_{\phi}$ with the local $\mathrm{T}_{\mathrm{i}}$ is clear. The line drawn at a slope of $45 \mathrm{~km} / \mathrm{s} / \mathrm{keV}$ is the scaling value described in reference [34] for unidirectional co-I $\mathrm{I}_{\mathrm{p}} \mathrm{NBI}$ in DIII-D, with the DIII-D NBI $\mathrm{D}^{+}$beams at $60 \mathrm{keV}$ for these data 
in Figure 2. The lines drawn at 18 and $10 \mathrm{~km} / \mathrm{s} / \mathrm{keV}$ will be related to the Parra scaling [10] discussed in Section VII.

The data used to establish and compare with the functional intrinsic scaling, equation (1), are mostly from $\mathrm{H}$-mode discharge conditions. However there are some L-mode conditions and even some with only Ohmic heating, although a very small minority. Additionally, the JET data used in Section V are L-mode conditions. A commonality for the dataset is that most all are with non-negligible auxiliary heating, that is, with a level near or above the Ohmic heating level.

\section{Dimensionless Intrinsic Rotation Scaling in DIII-D}

\section{A. Balanced NBI blip measurement and low injected torque}

The toroidal re-aiming of a neutral beamline has allowed a significant variation of the NBI injected torque in DIII-D discharges. One benefit for intrinsic rotation experiments has been that torque-balanced NBI blips can be used in intrinsic rotation experiments, greatly increasing the number of measurement times attainable in a single shot. The majority of the DIII-D data that are used to develop this dimensionless scaling law came from an experiment that scanned various discharge parameters within each shot with multiple "balanced-blips".

Time traces for a typical discharge in this dataset are shown in Figure 3. The delivered power is shown in Figure 3(a). The ECH power is 100\% absorbed within the plasma at the second harmonic resonance, as determined by the Toray-GA code [36]. The NBI power is mostly absorbed, but there is a small amount of shine-through and not all NBI ions thermalize before being lost. The NBI power is delivered in a series of blips, which become very dense later in the discharge as NBI power replaces the ECH power. The NBI injected torque is shown in Figure 3(b). The black traces are the total neutral beam toroidal momentum flux injected through the ports. The blue traces are smoothed by 25 msec to understand the minimal net torque injected by the isolated blips, and the small 
torque excursions that are programmed in the second phase. A nominal instantaneous injected torque for the more tangential DIII-D beams is $\sim 1 \mathrm{Nm} / \mathrm{MW}$ [34], so these torque levels are relatively small for DIII-D. The CER measured $V_{\phi}$ at $\rho=0.77$ is shown by the (red) circles in Figure 3(b). The ECH power is used to create the H-mode indicated by the sharp density rise at $\mathrm{t} \sim 1000 \mathrm{~ms}$ in Figure 3(c). The drop in density throughout the $\mathrm{ECH}$ phase is due to the well-known ECH "pumpout" effect [37], and reverses when the ECH power is turned off. There is a programmed step down in Ip to change the $\mathrm{q}_{95}$ value at $\mathrm{t} \sim 2500 \mathrm{~ms}$, and the value of $\beta_{\mathrm{N}}$ is also shown which changes with the changing discharge parameters. After a brief ELM-free phase following the L-H transition, the discharge exhibits type 1 ELMs [38] at a higher rate in the ECH phase than in the NBI power phase.

What we mean by nonperturbative torque from the blips is indicated in Figure 4(a), where we focus on two of the multi-beam blips shown in Figure 3(b). Here, the dashed curves show the injected torque again, with predominantly two co- $\mathrm{I}_{\mathrm{p}}$ directed beams followed by a counter- $\mathrm{I}_{\mathrm{p}}$ beam to null out the total torque impulse. The red traces are the total torque delivered to the plasma calculated with the TRANSP NUBEAM code [39]. In these conditions we calculate the neutral beam fast ion slowing time to be $\sim 50 \mathrm{msec}$, and the total energy confinement time to be $\sim 90 \mathrm{msec}$. Both of these times are short compared with the inter-pulse time of $400 \mathrm{msec}$ here, so there is very little effect of one blip on the next and the conditions remain "intrinsic".

There is some modulation of the plasma velocity profile during the high power blips, but we typically use only the first 1 or $2 \mathrm{msec}$ from a blip to specify the intrinsic conditions. In Figure 4(a) the total impulse from a blip is $\sim-0.005 \mathrm{~N}-\mathrm{m}-\mathrm{s}$, while the total angular momentum of the plasma is $\sim 0.1 \mathrm{~N}-\mathrm{m}-\mathrm{s}$, so on average we would expect roughly a $5 \%$ reduction in $\mathrm{V}_{\phi}$ from the blip. The net change is small, and has dissipated by the time of the next blip. We have verified this by halving the inter-pulse time with no change in the velocity profiles measured by the first msec of the blips in steady conditions. 
The ECH deposition location is shown in Figure 4(b), near $\rho=0.6$. Power from 5 gyrotrons at $110 \mathrm{GHz}$ is absorbed at the second harmonic of the electron cyclotron frequency, as indicated. The antennas are aimed to minimize any electron cyclotron current drive. At this time in the discharge $(2000 \mathrm{msec})$ the deposition is near the $\mathrm{q}=3 / 2$ surface.

\section{B. Empirical Scaling}

The scaling that emerged from this dataset is that

$$
\mathrm{M}_{\mathrm{A}}=\mathrm{C}_{\rho} \beta_{\mathrm{N}} \rho^{*}
$$

where $\mathrm{C}_{\rho}$ is a constant near unity. Here we use the standard "non-MKS" definition of $\beta_{\mathrm{N}} \equiv \beta \mathrm{aB} / \mathrm{I}_{\mathrm{p}}$, with $\mathrm{I}_{\mathrm{p}}$ in MA and $\beta$ is the global (averaged) quantity expressed in percent. The local value of toroidal velocity is used in $\mathrm{M}_{\mathrm{A}}$, as is the local $\mathrm{T}_{\mathrm{i}}$ in $\rho^{*} \equiv \mathrm{r}_{\mathrm{L}} / \mathrm{a}=\sqrt{2} \overline{\mathrm{v}} / \mathrm{a} \omega_{\mathrm{C}}$ with $\omega_{\mathrm{C}}=\mathrm{ZeB}_{0} / \mathrm{M}_{\mathrm{i}}, \mathrm{Z}$ the ion charge number and e the electron charge magnitude. In these normalizations, $\mathrm{V}_{\mathrm{A}}$ and $\beta_{\mathrm{N}}$ are independent of minor radius at each timeslice, and the only minor radial variation on the rhs is $\rho^{*} \sim \sqrt{T_{i}}$, for each timeslice.

A plot of $M_{A}$ versus $\beta_{N} \rho^{*}$ at a single minor radius location for this dataset is shown in Figure 5. The diagonal unity slope line is simply to guide the eye. The location in minor radius is the CER channel near $\rho=0.8$, that is, near the "peak" intrinsic velocity location that came out of the original Rice Scaling in DIII-D (Figure 1). The (red) solid circles are data for only the very first time measurement slice in an entire shot, before any other beam input. The (blue) open squares are from the first time measurement in every NBIblip in the ECH phase of a shot, as in Figure 3(a) prior to $t=4000 \mathrm{~ms}$, with the sparse blips. The cross symbols include every measurement time in an entire shot, both throughout the blip and the essentially steady NBI phase of a shot. The axes are logarithmic. Just over an order of magnitude is achieved for each axis in this correlation. In relation to the scaling $\mathrm{M}_{\mathrm{A}} \sim \beta_{\mathrm{N}}^{\mathrm{M}}[31]$ the $\rho *$ term has largely compensated for the $40 \pi$ numerical difference in magnitude between $\beta_{\mathrm{N}}^{\mathrm{M}}$ and $\beta_{\mathrm{N}}$. 
Using equation (1) to describe this set gives $\mathrm{C} \rho=1.25$, the value taken from fitting to the red points. As we show other data on this same graphical grid, a linear relationship between the variables will be reinforced. We shall use this $\mathrm{C} \rho$ value in comparing this scaling with the Rice and Parra scalings. If we constrain a fit to a power law for the abscissa and do a least squares fit to all the (blue) square points in Figure 5 we find that $\mathrm{M}_{\mathrm{A}}=\mathrm{a}_{\rho}\left(\beta_{\mathrm{N}} \rho^{*}\right)^{\mathrm{b}}$ with $\mathrm{a}_{\rho}=0.25+/-0.04$ and $\mathrm{b}=0.72+/-0.04$, shown by the dashed line.

This fit with the exponent $b$ less than unity is accommodating the lower $\beta_{\mathrm{N}} \rho^{*}$ data points in Figure 5, some of which are from L-mode conditions.

In figure 6(a) we show this scaling over a full radial profile rather than in the single outer radial location, where we plot the ratio $\mathrm{M}_{\mathrm{A}}(\rho) /\left[\beta_{\mathrm{N}} \rho^{*}(\rho)\right]$ versus $\rho$. The (red) solid circles are the values averaged over all the (red) first slice outer radial location data indicated in Figure 5. The vertical lines indicate the standard deviations for the averaged values. Also shown in Figure 6(a) are the ratios for one particular shot, the (red) circle with the smallest $\mathrm{M}_{\mathrm{A}}$ value in Figure 5. The ratio does not deviate greatly from unity over the entire profile. The interior, $\rho<0.4$ shows effects of ECH depression of the velocity profile there, and the edge, $\rho>0.85$ shows more structure due to the boundary effects [19]. The vertical line shows the radial location for the points plotted in Figure 5.

This same ratio near unity holds even for times in these shots when the NBI is injected continuously with a relatively small nonzero torque value, the time for $\mathrm{t}>4000 \mathrm{~ms}$ in Figure 3(a). In Figure 6(b) we show the radial profile average for these times as open circles. The standard deviation indicators are larger due to the torque excursions about 0 . The (blue) solid circles here show the averaged ratio profile for all the first slice time data corresponding to the (blue) open squares in Figure 5. The region near $\rho=0.8$ in Figure 6 (a) and (b) shows that the precise choice of this radial location is not critical.

For comparison we show in Figure 6(c) the outer radial profile of the same ratio for three times in a shot using the first blip measurements (blue squares in Figure 5) that are in an L-mode state, near the H-mode threshold, and have values toward the low end of the 
$\beta_{\mathrm{N}} \rho^{*}$ scale in Figure 5. In this shot, in a time sequence as shown in Figure 3, the ECH did not fire and only one CER beam functioned, providing measurements only in the radial location shown. Although not greatly different, the ratios at the nominal minor radius are a bit higher than those shown in Figures 6(a) and 6(b). It may be that for Lmode a location further out in minor radius is a better choice to match the same scaling. At the times $=1400,1600$ and $1800 \mathrm{~ms}, \beta_{\mathrm{N}}=0.44,0.52$ and 0.48 , respectively.

\section{Added DIII-D data}

Here we add more DIII-D experimental data to the plot space identical to that used in Figure 5. These data also come from LSND discharge shapes in DIII-D, but with different details. First, we take the data set that led to the Rice Scaling in DIII-D [4], and that were used in reference [10]. At the time these experiments were done all DIII-D beams were toroidally unidirectional and we used a single timeslice from the first beam blip in a discharge. Using these discharges in Figure 7 we plot $M_{A}$ versus $\beta_{N} \rho^{*}$ at the outer "peak" location, $\rho=0.78$, indicated in Figure 1, again following the general trend of unity slope.

Experiments using helium as the bulk ion were also performed in DIII-D and a similar plot from these discharges is shown in Figure 8. Here, the $\mathrm{He}^{2+}$ bulk ion measured values for $\mathrm{V}_{\phi}$ and $\rho^{*}$ are used in plotting the (red) solid circles. The value of $\rho^{*}$ is thus reduced by $1 / \sqrt{2}$ compared to that for $\mathrm{D}^{+}$at the same $\mathrm{T}_{\mathrm{i}}$ and $\mathrm{B}_{0}$. Discharges were run in pairs with CER measurements of the trace $\mathrm{C}^{6+}$ impurity done in one of the pairs, and these values are shown for the (blue) open squares. That is, for the $\mathrm{C}^{6+}$ measurements, the measured $V_{\phi}$ and $T_{i}$ are assigned to the bulk ion, helium, and used in the plots, just as we are doing for the deuterium main ion discharges.

In the intrinsic rotation similarity experiments in C-Mod and DIII-D, discharges were run in C-Mod to match DIII-D targeted conditions [4], and LSN discharges with the C-Mod shape were also run in DIII-D, with the unidirectional NBI injection in DIII-D. Scaling 
data taken from these discharges are shown in Figure 9 where we again plot $\mathrm{M}_{\mathrm{A}}$ versus $\beta_{\mathrm{N}} \rho^{*}$ on the same logarithmic axes. The (red) closed circles are from the discharges with the C-Mod shape, and the (blue) closed circles are from the same day where we enlarged the plasma cross section to match better with the typical DIII-D LSN shape that we had used in the original intrinsic rotation experiments.

The various shapes used in these data sets are indicated in Figure 10. In all discharges the minor radial location at or just inside of $\rho=0.8$ has been used. The (red) shape, "balanced blip", is the one used for the data plotted in Figure 5. The dotted shape is representative of those used in the original DIII-D data, as plotted in Figures 7 and 8. For the earliest of these experiments the lower baffle in DIII-D had not been extended, as indicated by the dotted boundary surface in that region. The (blue) shape, "C-Mod", is for the (red) closed circles in Figure 9 and the fourth (green) shape for the (blue) closed circles in Figure 9. All of these shapes result in a similar level of agreement with the scaling of equation (1), so if the details of the LSN shape matter for this scaling in some way, it would appear to be at a level that is within the scatter of the data points.

\section{C-Mod data and JET data}

The scaling data presented thus far have all been acquired in DIII-D experiments. Now we add intrinsic rotation data from discharges in C-Mod and JET that have previously appeared in publications. We take the four ICRF-heated discharge conditions in C-Mod that were extensively analyzed in the similarity comparison experiments [4]. In C-Mod, these ion velocity and temperature measurements are passive [21] and have no NBI torque injection associated with them. As in the original comparison between DIII-D and C-Mod (Figure 1), for C-Mod we used the on-axis velocity measurement, and $\mathrm{Ti}$ measurement, and we plot $\mathrm{M}_{\mathrm{A}}$ versus $\beta_{\mathrm{N}} \rho^{*}$ at this location in Figure 11 with open circles above the "C-Mod" call-out, using once again the same axes as the plots above. These symbols are color coded (online) to represent the four C-Mod discharges listed in Table 1 of reference [4], with the sequential shot numbers of that day shown on Figure 11. The 
two shots at larger $M_{A}(10,17)$ have $B_{0}=3.75 T$ and for the other two $(28,30) B_{0}=5.63$ $\mathrm{T}$.

For JET we use data from one of the first intrinsic rotation experiments done on JET, with results published in [9]. In JET short NBI pulses are used for CER velocity measurements of $\mathrm{C}^{6+}$, as in DIII-D. All NBI is in the co- $\mathrm{I}_{\mathrm{p}}$ direction for these discharges. Auxiliary heating in these JET conditions also is by ICRF. The intrinsic toroidal velocity profiles are similar to the ECH heated velocity profiles in DIII-D, in that there is a peak in toroidal velocity at larger minor radius. For low power ICRF conditions in 6 JET LSN discharges we plot $\mathrm{M}_{\mathrm{A}}$ versus $\beta_{\mathrm{N}} \rho^{*}$ at a "peak" velocity location in minor radius, $\mathrm{r} / \mathrm{a} \sim 0.8$ in Figure 11 adjacent the "JET" call-out. The (black) cross-symbols indicate first CER timeslice data within a discharge, equivalent to the (red) solid circles in Figure 5.

It has been noted that intrinsic toroidal velocity in JET high power ICRF conditions is small compared with an expectation from the Rice Scaling, and can even reverse to be in the counter- $\mathrm{I}_{\mathrm{p}}$ direction [40]. We see this effect in that the toroidal velocity falls below the equation (1) scaling in the data from these JET discharges in Figure 11 with increased ICRF power later in the shots. In Figure 12 we plot the ratio $\mathrm{M}_{\mathrm{A}} /\left[\beta_{\mathrm{N}} \rho^{*}\right]$ at $\rho \sim 0.8$ versus time for JET pulse 51664. Also shown are the ICRF power level as it is raised from 2 to $8 \mathrm{MW}$, and the value of $\beta_{\mathrm{N}}$. As the power is raised $\mathrm{M}_{\mathrm{A}} /\left[\beta_{\mathrm{N}} \rho^{*}\right]$ decreases significantly. It has been suggested that this may be caused by an effect due to ICRF fast ion loss [40]. In comparison with C-Mod, which is predominantly ICRF heated, we expect that the electron density values obtained in the four C-Mod discharges plotted in Figure 11 , all above $20 \times 10^{19} / \mathrm{m}^{3}$, are sufficient to attenuate fast minority ion tail formation [41].

\section{Relation to Rice Scaling}

We have shown some utility in the scaling given by equation (1) for intrinsic rotation in DIII-D, and for some discharges taken from C-Mod. Since both of these tokamaks have found evidence for a Rice scaling of toroidal velocity, it is natural to ask how equation 
(1) relates to Rice scaling. Numerically, for conversion from equation (1), we use the dimensionless $\beta_{\mathrm{N}}^{\mathrm{M}}$ that is numerically related to $\beta_{\mathrm{N}}$ by $\beta_{\mathrm{N}}=40 \pi \beta_{\mathrm{N}}^{\mathrm{M}}$. We define the volume averaged $\beta$ as $\beta=\left(\overline{\mathrm{p}}_{\mathrm{e}}+\overline{\mathrm{p}}_{\mathrm{i}}\right) /\left(B_{0}^{2} / 2 \mu_{0}\right)$ where the overbars indicate a volume average, and $\mathrm{p}=\mathrm{nT}$. To relate $\beta$ to the plasma thermal stored energy, $\mathrm{W}$, we use $\mathrm{W}=(3 / 2)\left(\overline{\mathrm{p}}_{\mathrm{e}}+\overline{\mathrm{p}}_{\mathrm{i}}\right) \mathrm{Vol}$, where $\mathrm{Vol}$ is the volume of the plasma. Using these relations in equation (1) we obtain

$$
\mathrm{V}_{\phi}(\mathrm{km} / \mathrm{s})=\mathrm{C}_{\rho}\left(\mathrm{W} / \mathrm{I}_{\mathrm{p}}\right)\left[5300 \sqrt{\mathrm{T}_{\mathrm{i}}(\mathrm{keV}) / \mathrm{n}_{\mathrm{e} 19}} /\left(\mathrm{B}_{0} \mathrm{Vol}\right)\right]
$$

and we see some added dependencies to a Rice-like scaling, specifically $\left(\mathrm{T}_{\mathrm{i}} / \mathrm{n}_{\mathrm{e}}\right)^{1 / 2} /\left(\mathrm{B}_{0} \mathrm{Vol}\right)$. Here we have defined $\mathrm{n}_{\mathrm{e} 19}=\mathrm{n}_{\mathrm{e}} / 10^{19}$ and taken a single ion species, so $n_{e}=Z_{i} n_{i}$.

In Figure 13 we plot the ratio for DIII-D data, $R_{D} \equiv V_{\phi}(k m / s) I_{p}(A) / W(J)$ versus $\left[\mathrm{T}_{\mathrm{i}}(\mathrm{keV}) / \mathrm{n}_{\mathrm{e} 19}\right]^{1 / 2}$ for the same data points shown as blue squares in Figure 5. The horizontal red line shows the mean value of $R_{D}=65$, and the dashed red lines show the standard deviation. The Rice scaling slope (that is, $\mathrm{R}_{\mathrm{D}}$ ) found in the original DIII-D dataset is 63 [4], very consistent with this dataset from the balanced blip era in DIII-D. Given the scatter in the original data plot there is ample room for added dependencies. The dashed line in Figure 13 shows the result of a simple least squares fit for the data, resulting in $\mathrm{R}_{\mathrm{D}}=193\left(\mathrm{~T}_{\mathrm{i}} / \mathrm{n}_{\mathrm{e} 19}\right)^{1 / 2}-2$.

For this dataset in Figure 13 the average value of BVol is $31 \mathrm{~T}-\mathrm{m}^{3}$. Using this in equation (2), $\mathrm{Z}_{\mathrm{i}}=1$, and $\mathrm{C}_{\rho}=1.25$ we obtain $\mathrm{R}_{\mathrm{D}}=214\left(\mathrm{~T}_{\mathrm{i}} / \mathrm{n}_{\mathrm{e} 19}\right)^{1 / 2}$, in reasonable agreement with the fit to the data points. In other words, there is consistency between the data and the definitions that led from equation (1) to equation (2) and so we propose that an improvement to the $\mathrm{W} / \mathrm{I}_{\mathrm{p}}$ intrinsic velocity scaling in so-called engineering parameters would be obtained by including the other parameters as shown in equation (2). 
A closer look at the kinetic profiles for two discharges with significant differences in $M_{A}$ from Figure 5 shows that the added terms may tend to have a relatively lesser change with changes in $\mathrm{W} / \mathrm{I}_{\mathrm{p}}$. These are the two conditions shown by the larger symbols in Figure 5. The parameters for these two discharges at these times are given in Table 1. The condition with $\beta_{\mathrm{N}}=1.18$ (larger $\left.\mathrm{M}_{\mathrm{A}}, 133046.2000\right)$ is at a time well into the $\mathrm{H}$-mode phase with steady ELMs, while the condition with $\beta_{\mathrm{N}}=0.51$ (smaller $\mathrm{M}_{\mathrm{A}}, 133051.1200$ ) is $100 \mathrm{~ms}$ after the $\mathrm{H}$-mode transition, during the subsequent density rise. The temperature and density profiles for these two cases are shown in Figure 14, (a) $T_{e}$, (b) $T_{i}$, (c) $n_{e 19}$, and $Z_{\text {eff, }}$ and (d) $T_{i} / n_{e 19}$. Fully stripped carbon is the only impurity used in the $Z_{\text {eff }}$ calculation. While there are significant differences in the density and temperature profiles, the profile of $T_{i} / n_{e 19}$ changes very little. The Rice scale values for these two cases, 65 and 58 in Table 1, are both close to the DIII-D average value, consistent with the same $T_{i} / n_{e 19}$ factor entering into equation (2). The value of $B_{0}$ changes by a factor of 1.36 between for these two cases, which is larger than the factor of 1.1 in the Rice scale values. So there would seem to be some adjustment also in $\mathrm{V}_{\phi}$ that compensates for $\mathrm{B}_{0}$ in equation (2). In conducting a scan of $\mathrm{W} / \mathrm{I}_{\mathrm{p}}$, at fixed plasma shape, one could change heating power, or the density with gas puffing, or simply operate at a different $\mathrm{I}_{\mathrm{p}}$. We would generally not expect the direct changes in the kinetic profiles, and the indirect changes due to changes in confinement, to leave $T_{i} / n_{e 19}$ constant at the radial location found for the Rice scaling in DIII-D. However, perhaps the change in this ratio, especially the square root, at least is in general smaller than the change in $\mathrm{W} / \mathrm{I}_{\mathrm{p}}$.

Table 1

\begin{tabular}{|l|l|l|l|l|l|l|l|l|l|l|l|l|}
\hline Shot.Time & $\boldsymbol{\beta}_{\mathbf{N}}$ & $\boldsymbol{\rho}^{*}$ & $\mathbf{M}_{\mathbf{A}}$ & $\mathbf{q 9 5}$ & $\mathbf{B}_{\mathbf{0}}(\mathbf{T})$ & $\mathbf{I}_{\mathbf{p}}(\mathbf{M A})$ & $\boldsymbol{\beta}(\boldsymbol{\%})$ & $\mathbf{V}_{\mathbf{\phi}(\mathbf{(}}(\mathbf{0 . 8})$ & $\mathbf{T}_{\mathbf{i}}(\mathbf{k e V})$ & $\mathbf{P}_{\mathbf{E C}}(\mathbf{M W})$ & $\mathbf{P}_{\mathbf{O H}}(\mathbf{M W})$ & $\mathbf{R}_{\mathbf{D}}$ \\
\hline 133046.2000 & 1.18 & .0057 & .0064 & 3.3 & 1.59 & 1.2 & 1.4 & 21.6 & 0.74 & 2.9 & 0.5 & 65 \\
\hline 133051.1200 & 0.51 & .0034 & .0021 & 5.1 & 2.16 & 1.0 & 0.4 & 11.8 & 0.48 & 1.3 & 1.0 & 58 \\
\hline
\end{tabular}

There is also an indication of the $1 / \mathrm{B}_{0}$ term in equation (2) in the C-Mod data used in Figure 11. For these data there are two values of $\mathrm{B}_{0}$ [4], having a ratio of 1.5 , which gives somewhat of a greater change than in the DIII-D data above. In figure 15 we plot the Rice scale ratio for C-Mod $R_{C} \equiv V_{\phi}(k m / s) I_{p}(A) / W(J)$ versus $B_{0}$ using the same data 
point conditions as in Figure 11. The dashed line shows a $1 / \mathrm{B}_{0}$ variation that is consistent with the data, given the spread.

In comparing the Rice scaling in C-Mod with that in DIII-D we had surmised that there should be a $1 /$ size $^{2}$ term multiplying $\mathrm{W} / \mathrm{I}_{\mathrm{p}}$. Indeed, we plot such a scaling in Figure 15 by the horizontal chain-dash line, showing $\left(a_{D} / a_{C}\right)^{2} R_{D}$, where $a_{D}=0.60 \mathrm{~m}$ and $a_{C}=0.22 \mathrm{~m}$ are the minor radii of DIII-D and C-Mod, respectively, as used in the similarity experiment [4]. This value agrees reasonably well with the C-Mod $\mathrm{R}_{\mathrm{C}}$. However, the $1 /$ Volume term in equation (2) points to $1 / \mathrm{size}^{3}$ for the Rice Scaling. The difference of course is the $\mathrm{B}_{0}$ term. In the DIII-D to C-Mod similarity comparisons $\mathrm{B}_{0}$ a is roughly constant, leaving only a $1 / \mathrm{a}^{2}$ variation in equation (2) for the comparison.

\section{Relation to Parra Scaling.}

The Parra Scaling (PS) for intrinsic rotation predicts a velocity proportional to $T_{i}$, $\mathrm{V}_{\phi}(\mathrm{km} / \mathrm{s})=\mathrm{C}_{\mathrm{P}} \mathrm{T}_{\mathrm{i}}(\mathrm{keV}) / \mathrm{I}_{\mathrm{p}}(\mathrm{MA})$, where $\mathrm{C}_{\mathrm{P}}$ is a constant [10]. The experimentally measured correlation of $\mathrm{V}_{\phi}$ with $\mathrm{T}_{\mathrm{i}}$ as shown in Figure 2 indicates that we should determine how our empirical scaling compares with this. PS focuses upon the interior region in minor radius and addresses inverted, i.e. hollow, velocity profiles, where $\mathrm{dV}_{\phi} / \mathrm{d} \rho>0$. In contrast, we here focus upon the outer region in minor radius having $\mathrm{dV}_{\phi} / \mathrm{d} \rho<0$. Nevertheless, the scaling given by equation (1) is of a similar magnitude to that given by PS.

To make this comparison we rewrite equation (1) by replacing $\mathrm{V}_{\mathrm{A}}$ by $\overline{\mathrm{V}}$, using $\mathrm{V}_{\mathrm{A}}=\overline{\mathrm{V}} / \sqrt{\beta_{\mathrm{i}} / 2}$, where $\beta_{\mathrm{i}}=\overline{\mathrm{p}}_{\mathrm{i}} /\left(\mathrm{B}_{0}^{2} / 2 \mu_{0}\right)$. Using this and the definition of $\rho^{*}$ in equation (1) leads to

$$
\mathrm{V}_{\phi}=\mathrm{C}_{\rho}\left(\frac{80 \pi}{\mu_{0} \mathrm{I}_{\mathrm{p}}}\right) \frac{\mathrm{T}_{\mathrm{i}}(\mathrm{eV}) \beta}{\mathrm{Z}_{\mathrm{i}} \sqrt{\beta_{\mathrm{i}}}}
$$


Using $Z_{i}=1$ and the same units following eqn (3) of Parra et al [10] we find

$$
\mathrm{V}_{\phi}(\mathrm{km} / \mathrm{s})=200 \mathrm{C}_{\rho}\left(\frac{\beta}{\sqrt{\beta_{\mathrm{i}}}}\right)\left(\frac{\mathrm{T}_{\mathrm{i}}(\mathrm{keV})}{\mathrm{I}_{\mathrm{p}}(\mathrm{MA})}\right)
$$

In the condition that $\mathrm{T}_{\mathrm{e}}=\mathrm{T}_{\mathrm{i}}$, so $\beta_{\mathrm{e}}=\beta_{\mathrm{i}}=\beta / 2$, and the value $\mathrm{C}_{\rho}=1.25$, we obtain $\mathrm{V}_{\phi}(\mathrm{km} / \mathrm{s})=36 \sqrt{\beta(\%)} \mathrm{T}_{\mathrm{i}}(\mathrm{keV}) / \mathrm{I}_{\mathrm{p}}(\mathrm{MA})$. In the Parra equation there is no $\beta$ term. The coefficient in the PS fit to experimental data is $C_{P}=18+/-4$, while the value based on their physical model is 10 [10]. The experimental data used in [10] included core data from the DIII-D discharges with outer location data shown in figure 7. In Figure 2 we have plotted these two values of $C_{P}, 18$ and 10 , with $I p=1 \mathrm{MA}$, a roughly median value for the $T_{i}$ dataset, to show the applicability to the lower $V_{\phi}$ side of the data, i.e. intrinsic conditions.

The added $\beta$ terms in our equations (3) and (4) may be indicators of the strength of the saturated turbulence that in conjunction with neoclassical orbit shifts is driving a radial momentum flux. In our introduction of $\beta_{\mathrm{i}}$ to switch to $\overline{\mathrm{V}}$ as the scaling velocity we have used the global $\beta_{\mathrm{i}}$ value since the $\beta_{\mathrm{N}}$ values we use are global. This results in the linear $T_{\mathrm{i}}$ dependence in equations (3) and (4). Had we used a local $T_{i}$ in $\beta_{i}$, our conversion would effectively result in a $\mathrm{T}_{\mathrm{i}}^{3 / 2}$ dependence.

We use equation (4) to compute a modified Parra Scaling (MPS) for the toroidal velocity profile, $V_{M P S}$, using experimental data. This means that the $V_{\text {MPs }}$ profile is the $T_{i}$ profile scaled as in equation (4). In computing $\beta_{i}$ we use the ion density given by $n_{i}=n_{D^{+}}+n_{C^{6+}}$. In Figure 16 (a) and (b) we show the MPS velocity profiles for the two discharge conditions described in TABLE 1 and Figure 14, together with the measured CER $\mathrm{C}^{6+}$ toroidal velocity (red). The dashed line in Figure 16(a) shows a vertically shifted $\mathrm{V}_{\text {MPS }}$ to compare the profile shapes in the outer region, our focus, while in Figure 16(b) no shift appears to be required. The PS does not specify the boundary condition. We also show the profiles of $\mathrm{V}_{\phi}(\mathrm{km} / \mathrm{s}) \equiv \mathrm{V}_{\mathrm{PS}}=18 \mathrm{~T}_{\mathrm{i}}(\mathrm{keV}) / \mathrm{I}_{\mathrm{p}}(\mathrm{MA})$ found in [10], and two shifted profiles for these. In the outer radial region of these two discharge conditions the scalings shown 
are of the correct magnitude and match the profile shape of the measured $V_{\phi}$ to varying agreement in the range $0.6<\rho<0.9$. The dip in measured $\mathrm{V}_{\phi}$ just inside the boundary at

$\rho=1$ is often seen in edge velocity measurements of $C^{6+}[42,19]$, and now more strongly in the main ion $\mathrm{D}^{+}$in DIII-D [14-16], where it is clearly related to a main ion edge flow layer. As noted, the depression in measured $V_{\phi}$ approaching the magnetic axis is induced by ECH, likely elevated $T_{e} / T_{i}$. In this region, $d V_{\phi} / d \rho>0$, the original PS is indicated, with $\mathrm{C}_{\mathrm{P}} \sim 11$ and 13, for Figure 16(a) and (b), respectively

PS is arrived at using arguments regarding the structure of the momentum transport equation in an expansion of the (small) parameter $\mathrm{r}_{\theta} / \mathrm{L}_{\mathrm{T}}$ and the ansatz that $\mathrm{L}_{\phi} \sim \mathrm{L}_{\mathrm{T}}$ [10]. $\quad$ Here, $\quad \mathrm{r}_{\theta} \equiv\left(\mathrm{B} / \mathrm{B}_{\theta}\right) \mathrm{r}_{\mathrm{L}}, \quad \mathrm{L}_{\mathrm{T}}^{-1} \equiv-\mathrm{RB}_{\theta}\left(\ln \mathrm{T}_{\mathrm{i}}\right)^{\prime}, \quad \mathrm{L}_{\phi}^{-1} \equiv-\mathrm{RB}_{\theta}\left(\ln \omega_{\phi}\right)^{\prime}, \quad \mathrm{f}^{\prime} \equiv \mathrm{df} / \mathrm{d} \psi$, $\omega_{\phi}=\mathrm{V}_{\phi} / \mathrm{R}, \mathrm{R}$ is the major radius, $\mathrm{B}_{\theta}$ is the poloidal magnetic field, and $\psi$ is the poloidal magnetic flux variable. The more general result in [10] appears as $\mathbf{V}_{\varphi} \sim\left(\mathbf{a} / \mathbf{L}_{\mathbf{T}}\right)\left(\mathbf{T}_{\mathbf{i}} / \mathbf{I}_{\mathbf{p}}\right)$ and it is assumed that $\left|\mathrm{L}_{T}\right| \sim$ a. While this is somewhat the case in the interior region, in the outer region of interest here we find that $\mathrm{a} / \mathrm{L}_{\mathrm{T}}$ varies significantly with radius, being well above 1. This could bring a different dependence on $T_{i}$ into the PS if applied in the outer region in minor radius.

\section{Added Torque}

In the latter phase of the discharges like those shown in Figure 3(b) there is a continuous addition of relatively small net NBI toroidal torque, changing directions in time. Here, in a global sense, we look at the effect of this torque on the scaling relation in equation (1). In Figure 17 we plot $\mathbf{M}_{\mathbf{A}} /\left(\boldsymbol{\beta}_{\mathrm{N}} \rho^{*}\right)$ versus the total injected NBI torque, smoothed backward in time by $50 \mathrm{msec}$. These are from all the times in a discharge beyond the application of continuous NBI. There is significant scatter in the data points, but a leastsquares linear fit gives a result that fits with other DIII-D experiments. The (red) dashed line is the least squares fit and using this fit to extrapolate, we find that a negative torque of $-1.93 \mathrm{~N}-\mathrm{m}$ results in $\mathrm{M}_{\mathrm{A}}=0$, that is, $\mathrm{V}_{\phi}=0$. This value is consistent with DIII-D experiments on zeroing out the intrinsic momentum with counter NBI torque [43]. The 
projected value here is a bit less, likely because it requires less counter torque to reduce the outer region $(\rho \sim 0.8)$ to 0 than the global plasma angular momentum to zero, as was done in reference [43].

\section{Summary}

Primarily using DIII-D data we have made the case for a dimensionless empirical scaling for the intrinsic toroidal velocity as given by equation (1). We apply this to the region in the vicinity of $\rho \sim 0.8$, away from the edge boundary condition and the gradients in velocity there, and away from the central core, where the (DIII-D) predominance of electron heating likely results in a hollowing of the otherwise co- $\mathrm{I}_{\mathrm{p}}$ toroidal velocity, due to a turbulent momentum stress that rearranges the velocity profile internally. Such a stress would be moving co-Ip momentum outward, thus tending to increase the co-Ip $\mathrm{V}_{\phi}$ in the vicinity of $\rho \sim 0.8$. Formerly, we found a better fit for DIII-D data to a Rice-like scaling by multiplying $\left(\mathrm{W} / \mathrm{I}_{\mathrm{p}}\right)$ by $\left(\mathrm{T}_{\mathrm{e}} / \mathrm{T}_{\mathrm{i}}\right)_{0}$, where the temperatures were taken from the core region [4]. Additionally $T_{e} / T_{i}$ enters our scaling if we look at the form given in equation (3), through $\sqrt{\beta / \beta_{i}}$. Beyond this we have not looked systematically for an effect of $\mathrm{T}_{\mathrm{e}}$, or $\mathrm{dT}_{\mathrm{e}} / \mathrm{d} \rho$, on the empirical scaling given here. Recently, gyrokinetic simulations have been used to calculate the core turbulent momentum stress driven by EC heating and obtain quantitative agreement with DIII-D hollow rotation profiles measured for the main ion population [44].

Avoiding the edge region in developing a scaling is also better for using the measured ambient impurity carbon velocity as a proxy for the main ion deuterium velocity; now with the DIII-D main ion CER system we measure large differences in the velocities of these two species close to the boundary in certain conditions [28]. To date, the main ion data that can be used to test equation (1) is limited and we will do further experiments obtaining main ion velocity profiles.

Given the range of variation measured in intrinsic rotation velocity profiles, we would not claim that the value given by equation (1) can be used to precisely predict a rotation 
velocity at $\rho=0.8$, but rather that this velocity is highly indicative of the magnitude of the velocity that will be realized in the vicinity of this minor radius, especially for $\mathrm{H}$ mode conditions. The agreement would be within a factor of 2 , given a cursory scan of the data scaling plots presented here. We emphasize that this is for reasonably good axisymmetric conditions, no large error fields, no applied non-axisymmetric fields, and only small internal broken symmetry, that is, MHD activity.

In addition to DIII-D data, we have included data from JET and C-Mod discharges that have been the subject of prior publication [4,9]. In JET, the L-Mode discharges had relatively low power ICRF heating and the velocity profiles are qualitatively similar to the ECH DIII-D discharges, so we used the same methodology in adding these data. For C-Mod, we use the same comparison rule as indicated in Figure (1), using a core velocity value in equation (1). The higher C-Mod densities, especially in H-mode, maintain $\mathrm{T}_{\mathrm{e}} \sim$ $\mathrm{T}_{\mathrm{i}}$ and one would not expect a hollow velocity profile in the discharge data used in Figure (11), at least not due to elevated $\mathrm{T}_{\mathrm{e}} / \mathrm{T}_{\mathrm{i}}$.

We have shown how the simple formula given in equation (1) relates to the Rice scaling for intrinsic rotation, $\mathrm{V}_{\phi} \sim \mathrm{W} / \mathrm{I}_{\mathrm{p}}$, a scaling which helped to motivate experiments targeting a universal explanation based on intrinsic plasma effects. As shown in equation (2), we now posit that the Rice scaling does not contain all the necessary terms, but apparently two of the more important terms.

The correlation of $\mathrm{V}_{\phi}$ with $\mathrm{T}_{\mathrm{i}}$ as shown in Figure (2), and described in other experiments $[10,18,32-34]$ is also captured in equation (1). Equation (1) can be cast in a form that reveals its relation to the PS [10], $\mathrm{V}_{\phi} \sim \mathrm{T}_{\mathrm{i}} / \mathrm{I}_{\mathrm{p}}$, as shown in equation (4), although we apply it to a region where the gradient in $\mathrm{V}_{\phi}$ is negative, rather than positive as done in [10]. To obtain this $T_{i}$ scaling we use a global $\beta_{i}$ rather than the local $T_{i}$ in this term. We call this a modified PS, modified by the addition of the $\beta$ terms, which do not appear in [10]. We show the comparison of the full $\mathrm{V}_{\phi}$ profile calculated by equation (4) with two measured 
$\mathrm{V}_{\phi}$ profiles from the dataset used here. The level of agreement varies, but the magnitude of the calculated profiles is very similar to that measured.

As we have shown, equation (1) can be cast in various forms, some likely more revealing than others. One form of equation (1) that may provide physical insight is also dimensionless, which we write as

$$
\mathrm{V}_{\phi}=\tilde{\mathrm{CS}} \frac{\beta}{\sqrt{\beta_{\mathrm{i}}}} \rho_{\theta}^{*} \overline{\mathrm{V}}
$$

where $\tilde{\mathrm{C}}$ is a constant, $\mathrm{S}$ a shape factor, and $\rho_{\theta}^{*}=\left(\mathrm{B} / \mathrm{B}_{\theta}\right) \rho^{*}$ is the poloidal gyroradius scaled to minor radius. $\mathrm{S}$ is the ratio of $\mathrm{B}_{\theta}$ to its value calculated for a cylinder. Equation (5) is nondimensional, if written for $\mathbf{M}_{\varphi} \equiv \mathbf{V}_{\phi} / \overline{\mathbf{V}}$, but we write it as in equation (5) to see the velocity dependence on $T_{i}$ explicitly, apart from the $\beta$ terms. This equation may indicate that an intrinsic momentum flux is being transported into the plasma by thermal ions sampling saturated turbulent fluctuations, driven by the $\beta$ terms, and the turbulent flows have the requisite broken symmetry on the scale of the drift centers varying by the poloidal ion gyroradius $[20,45]$.

Lastly, we make a projection using conditions calculated for the ITER tokamak as described for Inductive Scenario 1 of [46]. We take a $=2.0 \mathrm{~m}, \mathrm{~B}_{0}=5.3 \mathrm{~T},\left\langle\mathrm{n}_{\mathrm{e}}>=11\right.$ $\mathrm{x} 10^{19} / \mathrm{m}^{3}, \beta_{\mathrm{N}}=2.0$, and $\mathrm{T}_{\mathrm{i}}(\rho=0.8)=5 \mathrm{keV}$, and compute $\rho^{*}=\mathbf{. 0 0 1 3}$, and $\mathbf{V}_{\mathrm{A}}=8 \times 10^{6} \mathrm{~m} / \mathrm{s}$. Using equation (1) we obtain $\mathrm{V}_{\phi}=26 \mathrm{~km} / \mathrm{s}$, with $\mathrm{M}_{\mathrm{A}}=2.6 \times 10^{-3}$ $\left(\mathrm{D}^{+}\right)$. This should be taken as a being a prediction for the peak value for an ITER intrinsic velocity profile similar to those seen in Figure 16 for these ITER scenario parameters.

Notable in this ITER projection is the similarity of the magnitude of $\mathrm{V}_{\phi}$ in comparison with Figure 16, showing DIII-D data. Parra et al [10] noted that their scaling has no size dependence, but having the same velocity value would require a certain scaling for $T_{i}$ with $\mathrm{I}_{\mathrm{p}}$. This limited variation in the magnitude of intrinsic velocity can be understood if we simply use the modified Rice scaling, equation (2), together with the empirically 
determined H-mode confinement time, to project the stored energy with power, $\mathrm{W}=\tau_{\mathrm{E}-\mathrm{H}} \mathrm{P}$. We use $\tau_{\mathrm{E}-\mathrm{H}}=\mathrm{H} \tau_{\text {th }}$, where $\mathrm{H}$ is a usual $\mathrm{H}$-factor multiplier for the confinement quality and $\tau_{\mathrm{th}}=\tau_{\mathrm{th}, 04(2)}$ as given by equation (35), section 5.3.3 of [32]. Setting exponents less than $1 / 6$ equal to 0 to focus on the dominant terms, we arrive at $\mathrm{V}_{\phi}(\mathrm{km} / \mathrm{s})=630 \mathrm{H}\left[\sqrt{\mathrm{T}_{\mathrm{i}}(\mathrm{keV})} / \mathrm{B}_{0}\right][\mathrm{P}(\mathrm{MW}) / \mathrm{Vol}]^{1 / 2}\left[\mathrm{aR}^{2} / \mathrm{Vol}\right]^{1 / 2}$. Using the values for the DIII-D shot and time 133046, 2000ms, respectively, see Table 1 and Figure 14, we calculate that $\sqrt{\mathrm{T}_{\mathrm{i}}} / \mathrm{B}_{0}=\sqrt{0.74} / 1.60=0.54, \quad(\mathrm{P} / \mathrm{Vol})^{1 / 2}=(3.6 / 18.3)^{1 / 2}=0.44$, and $\left(\mathrm{aR}^{2} / \mathrm{Vol}\right)^{1 / 2}=\left(0.61 \times 1.72^{2} / 18.3\right)^{1 / 2}=0.32$, which leads to the prediction that $\mathrm{V}_{\phi}(\mathrm{km} / \mathrm{s})=48 \mathrm{H}$. In these $\mathrm{ECH}$ dominated discharges $\mathrm{H}$ is typically less than 1 , here we compute $\mathrm{H}=0.71$, so $\mathrm{V}_{\phi}=34 \mathrm{~km} / \mathrm{s}$. This is a reasonable value compared to the measured profile in Figure 16(a). Now for the ITER conditions we take $a / R=2 \mathrm{~m} / 6.2 \mathrm{~m}, B_{0}=5.3$, $\mathrm{T}_{\mathrm{i}}=5, \mathrm{P}=100 \mathrm{MW}$, and $\mathrm{Vol}=760 \mathrm{~m}^{3}$. The same values become, $\sqrt{\mathrm{T}_{\mathrm{i}}} / \mathrm{B}_{0}=0.42$, $(\mathrm{P} / \mathrm{Vol})^{1 / 2}=0.36$, and $\left(\mathrm{aR}^{2} / \mathrm{Vol}\right)^{1 / 2}=0.32$. None of these parameters differ greatly from the DIII-D values, in fact the product is smaller due to the smaller power per volume and $T_{i}$ term in this ITER scenario. This prediction for ITER gives $\mathrm{V}_{\phi}(\mathrm{km} / \mathrm{s})=31 \mathrm{H}$. Now, if we had kept the density term in the confinement scaling, $\mathrm{n}_{\mathrm{e} 19}^{-0.13}$, with $\mathrm{n}_{\mathrm{e} 19}=11$ in this ITER scenario, this brings the velocity prediction down to $\mathrm{V}_{\phi}(\mathrm{km} / \mathrm{s})=23$ with $\mathrm{H}=1$, consistent with our estimation from equation (1) where we obtained the stored energy value by using the ITER-given $\beta$. The methods are consistent to the extent that the energy confinement time scaling predicts the stored energy. In DIII$\mathrm{D}$ we needed to take a reduced value for $\mathrm{H}$, as obtained by comparing the actual stored energy with that calculated from [31]. Intrinsic rotation is energy dependent, so heating and confinement determine it. The $I_{p}$ scaling in energy confinement removes $I_{p}$ from the final equation for $\mathrm{V}_{\phi}$. As we go from small to large machine size we find a much smaller variation in the absolute value of the intrinsic $\mathrm{V}_{\phi}$, due to the practical limitations in auxiliary heating arising from cost, and the power degradation in energy confinement. 
We have arrived at two predictions for the "outer" region intrinsic toroidal velocity in ITER which are essentially equal given the scatter in the data. One is $26 \mathrm{~km} / \mathrm{s}$ based upon the projection from the dimensionless scaling equation (1) where the input is the ITER specified $\beta_{\mathrm{N}}, \mathrm{B}_{0}, \mathrm{~T}_{\mathrm{i}}$, average density, and minor radius a. The second is $23 \mathrm{~km} / \mathrm{s}$ where we use the engineering parameter scaling given in equation (2) with the terms from equation (1) that have converted this from the basic Rice scaling. However, to show the dominant terms affecting this velocity we utilized a standard H-mode confinement scaling for the stored energy, leading to cancelation of a number of terms, e.g. $I_{p}$, and then in addition to the above ITER specified parameters we needed the heating power density and a shape parameter. Whether this velocity is sufficient to keep ITER rotating in the direction of Ip is to be determined, given the level of non-axisymmetric fields in ITER, such as toroidal field ripple, magnetic perturbations for ELM suppression coils, and ferritic structures such as the Test Blanket Modules.

\section{Acknowledgments}

This material is based upon work supported by the U.S. Department of Energy, Office of Science, Office of Fusion Energy Sciences, using the DIII-D National Fusion Facility, a DOE Office of Science user facility, under Awards DE-FC02-04ER54698, DE-AC0209CH11466 ${ }^{2}$, and DE-FG02-04ER54235 $5^{3}$. DIII-D data shown in this paper can be

obtained in digital format by following the links at https://fusion.gat.com/global/D3D_DMP. Our thanks to the DIII-D team that made possible the acquisition and analysis of the vast amount of data that has been used in coming to the conclusions presented here. One of us (J. S. dG) acknowledges valuable discussions with R.E. Waltz. 


\section{References J deGrassie A28189}

1. Martin Greenwald, Phys. Plasmas 17, 058101, (2010).

2. Francis F. Chen, 2011, An Indispensible Truth: How Fusion Power Can Save the Planet, Springer, New York, p. 298.

3. C.C. Petty, Phys. Plasmas 15, 080501 (2008).

4. J.S. deGrassie, J.E. Rice, K.H. Burrell, R.J. Groebner, and W.M. Solomon, Phys. Plasmas 14, 056115 (2007).

5. B.P. Duval, A. Bortolon, A Karpushov, R.A. Pitts, A. Pochelon, A. Scarabosio, and the TCV Team, Plasma Phys. Control. Fusion 49, B195 (2007).

6. J.E. Rice A.C. Ince-Cushman, M.I. Reinke, Y. Podpaly, M.J. Greenwald, B. LaBombard, and E.S. Marmar, Plasma Phys. Control. Fusion 50, 124042 (2008).

7. K. Ida and J.E. Rice, Nucl. Fusion 54, 045001 (2014).

8. J.S. deGrassie, Plasma Phys. Control. Fusion 51, 124047 (2009).

9. J.-M. Noterdaeme, E. Righi, V. Chan, J. deGrassie, K. Kirov, M. Mantsinen, M.F.F. Nave, D. Testa, K.-D. Zastrow, R. Budny, et al, Nucl. Fusion 43, 274 (2003).

10. F.I. Parra, M.F.F. Nave, A.A. Schekochihin, C. Giroud, J.S. deGrassie, J.H.F. Severo, P. de Vries, and K.-D. Zasrow, Phys. Rev. Lett. 108, 095001 (2012).

11. P.H. Diamond, Y. Kosuga, Ö.D. Gürcan, C.J. McDevitt, T.S. Hahm, N. Fedorczak, J.E. Rice, W.X. Wang, S. Ku, J.M. Kwon, et al, Nucl. Fusion 53, 104019 (2013).

12. C. Angioni, R.M. McDermott, F.J. Casson, E. Fable, A. Bottino, R. Dux, R. Fischer, Y. Podoba, T. Pütterich, F. Ryter, et al, Phys. Rev. Lett. 107, 215003 (2011).

13. M. Barnes , F.I. Parra, J.P. Lee, E.A. Belli, M.F.F. Nave, and A.E. White, Phys. Rev. Lett. 111, 055005 (2013).

14. Z.X. Lu, W.X. Wang, P.H. Diamond, G. Tynan, S Ethier, C. Gao, and J. Rice, Phys. Plasmas 22, 055705 (2015).

15. J.A. Boedo, E.A. Belli, E. Hollmann, W.M. Solomon, D.L. Rudakov, J.G. Watkins, R. Prater, J. Candi, R.J. Groebner, K.H. Burrell, et al, Phys. Plasmas 18, 032510 (2011). 16. S.H. Mueller et al, Phys. Rev. Lett. 106, 115001 (2011).

17. J.A. Boedo, J.S. deGrassie, B. Grierson, T. Stoltzfus-Dueck, D.J. Battaglia, D.L. Rudakov, E.A. Belli, R.J. Groebner, E. Hollmann, C. Lasnier, et al, “Experimental 
Evidence of Edge Intrinsic Momentum Source Driven by Kinetic Ion Loss and Edge Radial Electric Fields in Tokamaks" Phys. Plasmas (submitted).

18. J.S. deGrassie, S.H. Müller, and J.A. Boedo, Nucl. Fusion 52, 013010 (2012).

19. J.S. deGrassie, R.J. Groebner, K.H. Burrell, and W.M. Solomon, Nucl. Fusion 49, 085020 (2009).

20. Janghoon Seo, C.S. Chang, S. Ku, J.M. Kwon, W. Choe, and Stefan H. Müller, Phys. Plasmas 21, 092501 (2014).

21. J.E. Rice, J.A. Goet, R.S. Granetz, M.J. Greenwald, A.E. Hubbard, I.H. Hutchinson, E.S. Marmar, D. Mossessia, T. Sunn Pedersen, J.A. Snipes, et al, Phys. Plasmas 7, 1825 (2000).

22. I.H. Hutchinson, J.E. Rice, R.S. Granetz, and J.A. Snipes, Phys. Rev. Lett. 84, 3330 (2000).

23. J.E. Rice, R.L. Boivin, P.T. Bonoli, J.A. Goetz, R.S. Granetz, M.J. Greenwald, I.H. Hutchinson, E.S. Marmar, G. Schilling, J.A. Snipes, et al, Nucl. Fusion 41, 277 (2001).

24. I.H. Hutchinson, R. Boivin, P.T. Bonoli, C. Boswell, R. Bravenec, N.L. Bretz, R. Chatterjee, T. Chung, E. Eisner, C. Fiore, et al, Nucl. Fusion 41, 1391 (2001).

25. J.S. deGrassie, K.H. Burrell, L.R. Baylor, W. Houlberg, and J. Lohr, Phys. Plasmas 11, 4323 (2004).

26. J.S. deGrassie, K.H. Burrell, L.R. Baylor, W.A. Houlberg, and W.M. Solomon, Proc. $20^{\text {th }}$ IAEA Fusion Energy Conf., Vilamoura, EX/6-4Rb (2004).

27. R.C. Isler, Plasma Phys. Control. Fusion 36, 171 (1994).

28. B.A. Grierson, K.H. Burrell, W.W. Heidbrink, M.J. Lanctot, N.A. Pablant, and W.M. Solomon, Phys. Plasmas 19, 056107 (2012).

29. C. Angioni, A.G. Peeters, X. Garbet, A. Manini, F. Ryter and ASDEX Upgrade Team, Nucl. Fusion 44, 827 (2004).

30. W.M. Solomon, K.H. Burrell, A.M. Garofalo, A.J. Cole, R.V. Budny, J.S. deGrassie, W.W. Heidbrink, G.L. Jackson, M.J. Lanctot, R. Nazikian, et al, Nucl. Fusion 49, 085005 (2009). 
31. J.E. Rice, A. Ince-Cushman, J.S. deGrassie, L.-G. Eriksson, Y. Sakamoto, A. Scarabosio, A. Bortolon, K.H. Burrell, B.P. Duval, C. Fenzi-Bonizec, et al, Nucl. Fusion 47, 1618 (2007).

32. E.J. Doyle, W.A. Houlberg, Y. Kamada, V. Mukhovatov, T.H. Osborne, A. Polevoi, G. Bateman, J.W. Connor, J.G. Cordey, T. Fujita, et al, Nucl. Fusion 47, S18 (2007).

33. J.E. Rice, J.W. Hughes, P.H. Diamond, Y. Kosuga, Y.A. Podpaly, M.L. Reinke, M.J. Greenwald, Ö.D. Gürcan, T.S. Hahm, A.E. Hubbard, et al, Phys. Rev. Lett. 106, $215001(2011)$.

34. J.S. deGrassie, D.R. Baker, K.H. Burrell, P. Gohil, C.M. Greenfield, R.J. Groebner and D.M. Thomas, Nucl. Fusion 43, 142 (2003).

35. P.C. de Vries, M.-D Hua, D.C. McDonald, C. Giroud, M. Janvier, M.F. Johnson, T. Tala, K.-D. Zastrow and JET EFDA Contributors, Nucl. Fusion 48, 065006 (2008). 36. Y.R. Lin-Liu, V.S. Chan, and R. Prater, Phys. Plasmas 10, 4064 (2003).

37. V. Erkmann and U. Gasparino, Plasma Phys. Control. Fusion 36, 1869 (1994).

38. A.W. Leonard, Phys. Plasmas 21, 090501 (2014).

39. A. Pankin, D. McCune, R. Andre, G. Batemann, and A. Kritz, Comput. Phys. Commun. 159, 157 (2004).

40. M.F.F. Nave, L.-G. Eriksson, C. Giroud, T.J. Johnson, K. Kirov, M.-L. Mayoral, J.-M. Noterdaeme, J. Ongena, G. Saibene, R. Sartori, et al, Plasma Phys. Control. Fusion 54, 074006 (2012).

41. A. Bader, R.S. Granetz, R.R. Parker, P.T. Bonoli, I.H. Hutchinson, J. Sears and S.J. Wukitch, Nucl. Fusion 52, 094019 (2012).

42. T. Pütterich, E. Wolfrum, R. Dux, and C.F. Maggi (ASDEX Upgrade Team), Phys. Rev. Lett. 102, 025001 (2009).

43. W.M. Solomon, K.H. Burrell, J.S. deGrassie, R. Budny, R.J. Groebner, J.E. Kinsey, G.J. Kramer, T.C. Luce, M.A. Makowski, D. Mikkelsen, et al, Plasma Phys. Control. Fusion 49, B313 (2007).

44. B.A. Grierson, W.X. Wang, S. Ethier, D.J. Battaglia, J.A. Boedo, J.S. deGrassie and W.M. Solomon, "Main-ion intrinsic rotation profile driven by residual stress torque from ion temperature gradient turbulence in the DIII-D tokamak", Phys. Rev. Lett. (submitted). 45. T. Stoltzfus-Dueck, Phys. Plasmas 19, 055908 (2012). 
46. B.J. Green Plasma Phys. Control. Fusion 45, 687 (2003). 


\section{Figure Captions}

Table 1. Parameters for the two discharge profiles plotted in Figure 14. These parameters are defined in the text.

Figure 1: Intrinsic toroidal Mach profiles in DIII-D and C-Mod showing the near equivalence of the maximum values. Reproduced with permission from J.S. deGrassie, J.E. Rice, K.H. Burrell, R.J. Groebner, and W.M. Solomon, Phys. Plasmas 14, 056115 (2007). Copyright (2007) American Institute of Physics.

Figure 2. $\mathrm{V}_{\phi}$ vs $\mathrm{T}_{\mathrm{i}}$ for all spatial and temporal measurements made for the DIII-D dataset used in Section 4. The slope of 45 is related to NBI driven conditions in DIII-D while those at 18 and 10 relate to the Parra Scaling described in Section VII.

Figure 3. (a) Power format for intrinsic rotation discharges. ECH power causes an $\mathrm{H}-$ mode transition, followed by balanced NBI blips for CER measurements, then ECH is replaced by NBI with nearly balanced NBI torque injection. (b) Torque injected by beam pulses into the torus. The (blue) trace is smoothed to show a time averaged value, with small sweeps to follow the plasma velocity response. $\mathrm{V}_{\phi}$ at an outer radial location is shown by the (red) circles. (c) Averaged electron density $\left(10^{19} / \mathrm{m}^{3}\right), \mathrm{q}_{95}, \beta_{\mathrm{N}}$, and $\mathrm{D}_{\alpha}$ divertor recycling light.

Figure 4. Auxiliary torque and EC deposition. (a) Balanced beam blips, instantaneous injected torque shown by the dashed line. TRANSP-computed torque absorbed by the plasma shown by the (red) line. (b) Flux surfaces for LSN shape with the deposition location for $\mathrm{ECH}$ power, at $\rho \sim 0.6$.

Figure 5. $\mathrm{M}_{\mathrm{A}}$ versus $\beta_{\mathrm{N}} \rho^{*}$ for intrinsic rotation conditions and small NBI torque conditions in DIII-D discharges with heating scenarios like that in figure 2 . Red is first timeslice in an entire discharge. Blue is first timeslice in sparse balanced beam blips. Others are all remaining measurement times in a discharge, some during small added net toroidal torque.

Figure 6. Radial profiles of the scaling taken from eqn (1), the vertical axis is $\mathrm{C} \rho$. (a) Solid (red) circles are the values averaged for the first time-slice (red) points shown in Fig. 5. The vertical bars show the standard deviations, while the vertical slice gives the average $\rho$ used for the points in Fig 5. The open circles are from one discharge time, the large (red) solid circle in Fig 5 (b) The (blue) solid circles show the averaged values for the (blue) squares in Fig 5, using the first slices from all the balanced NBI blips, and the open circles for all the times with steady NBI having times of nonzero NBI torque, the small crosses in Fig 5. (c) On different scales three time slices from L-mode conditions at low $\mathrm{M}_{\mathrm{A}}$ in Fig 5 are shown. The shaded vertical region shows the locations used for the points in Fig 5. 
Figure 7. Scaling plot for DIII-D data used in the original Rice-scaling experiments [4]. All of these points are taken from a single (first) blip timeslice in a discharge.

Figure 8. Scaling plot for DIII-D discharges having helium as the main ion. All of these points are taken from a single (first) blip timeslice in a discharge. The (red) solid circles are taken from measurements of $V_{\phi}$ for the helium bulk ion. The (blue) squares use $V_{\phi}$ measured for the trace $\mathrm{C}^{6+}$ impurity in these discharges.

Figure 9. Scaling plot for DIII-D discharges from a similarity experiment with C-Mod. The (red) solid circles utilize the scaled LSN C-Mod shape. The (blue) solid circles are with a larger volume LSN shape for comparison. All of these points are taken from a single (first) blip timeslice in a discharge.

Figure 10. Typical DIII-D LSN shapes used for the scaling data plotted in Fig 5 (red) "balanced blip", Fig. 7 and Fig. 8 (dotted), Fig. 9 (blue) for the "C-Mod" shape, and Fig. 9 (green) for the larger volume comparison.

Figure 11. Scaling plot for discharges taken from C-Mod and JET. The C-Mod data are from four discharges described in Table 1 of reference [4]. The JET data are from the low ICRF power phase of discharges presented in reference [9]. The black crosses in the JET points are from the first CER time slice in the NBI blips utilized there.

Figure 12. Decay of the scaling proportionality in a JET discharge with the increase in ICRF power.

Figure 13. Test of the eqn (1) scaling cast as a Rice-like scaling in eqn (2), using the data in Fig 5 shown as (blue) squares. The ratio $\mathrm{R}_{\mathrm{D}}$ is plotted versus $\left(\mathrm{T}_{\mathrm{i}}(\mathrm{keV}) / \mathrm{n}_{\mathrm{e} 19}\right)^{1 / 2}$

Figure 14 Profiles for the two discharge conditions represented by large symbols in Fig 5, with scaling parameters given in Table 1. (a) $\mathrm{T}_{\mathrm{e}}$ (b) $\mathrm{T}_{\mathrm{i}}$ (c) $\mathrm{n}_{\mathrm{e} 19}$ and $\mathrm{Z}_{\text {eff }}$ (carbon impurity) and (d) $\mathrm{T}_{\mathrm{i}} / \mathrm{n}_{\mathrm{e} 19}$.

Figure 15. C-Mod Rice-scale ratio, $\mathrm{R}_{\mathrm{C}}$, versus $\mathrm{B}_{0}$ using the $\mathrm{C}-$ Mod data from Fig. 11. The dashed line $\sim 1 / \mathrm{B}_{0}$. Minor radii are $\mathrm{a}_{\mathrm{D}}=0.60 \mathrm{~m}$ and $\mathrm{a}_{\mathrm{C}}=0.22 \mathrm{~m}$.

Figure 16. Modified Parra Scaling velocity $\mathrm{V}_{\mathrm{MPS}}$, computed with eqn (4) for the discharge conditions shown in Fig 14, and described in Table 1. The measured $V_{\phi}$ is shown (red). The dashed (blue) line shows $\mathrm{V}_{\mathrm{MPS}}$ rigidly shifted vertically to judge the conformity with $V_{\phi}$ where $d V_{\phi} / d \rho<0$. Also plotted is the fitted Parra value from [10] as $\mathrm{V}_{\mathrm{PS}}$, with similar shifts shown.

Figure 17. Response of the scaled $\mathrm{M}_{\mathrm{A}}$ to added net torque in the second phase of discharges like the example in Fig. 3. 


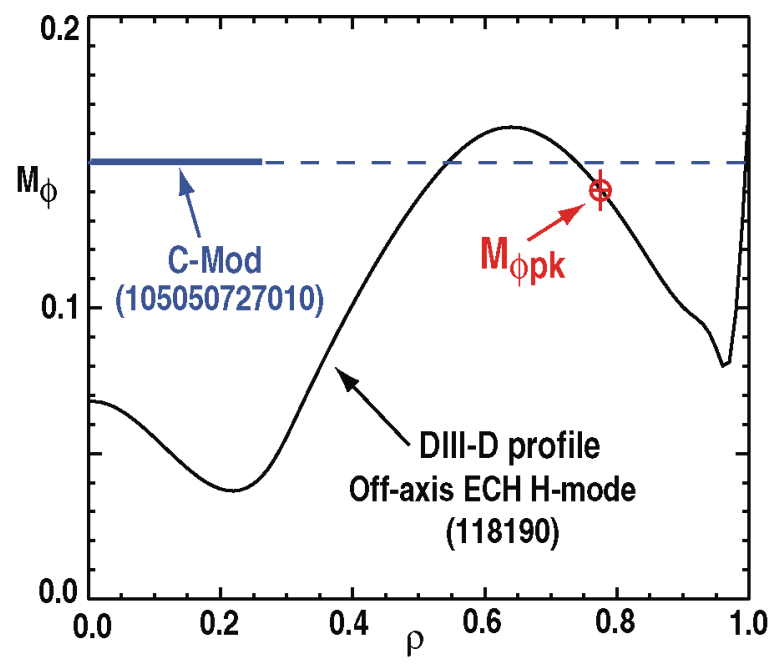

J.S. deGrassie Fig. 1 


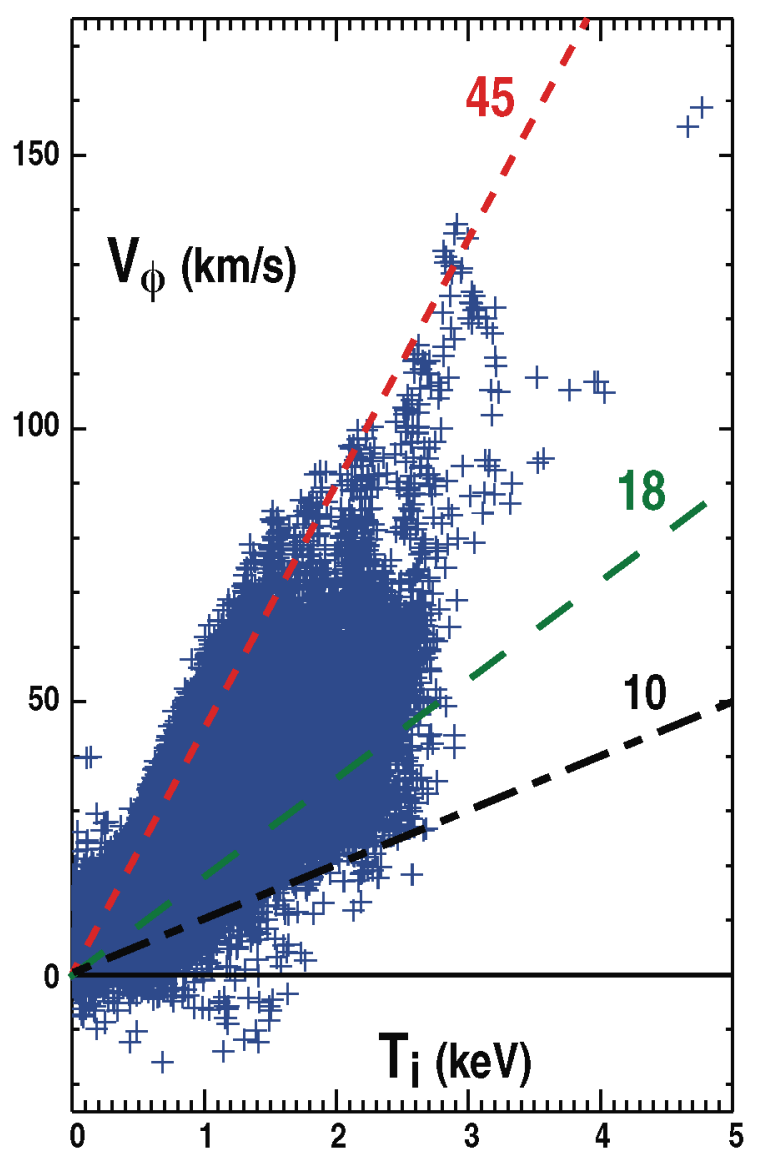

J.S. deGrassie Fig. 2 

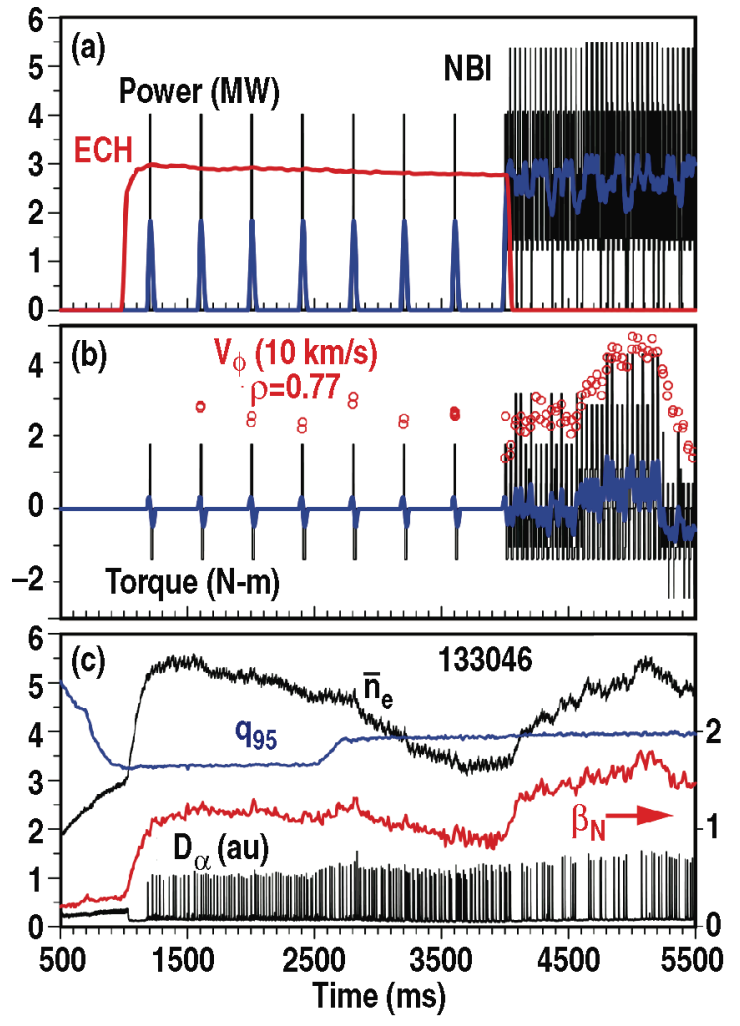

J.S. deGrassie Fig. 3 


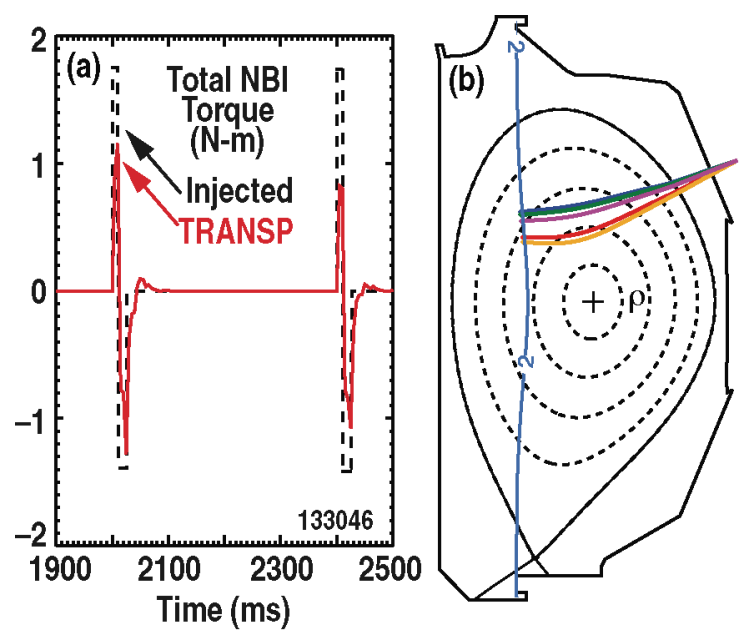

J.S. deGrassie Fig. 4 


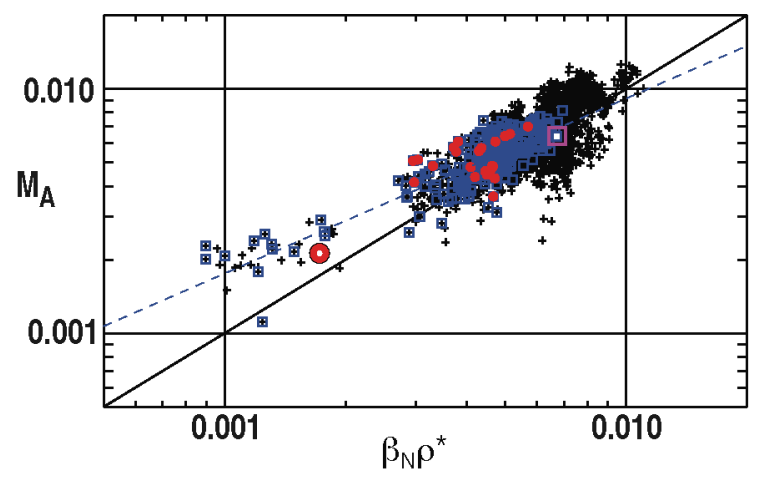

J.S. deGrassie Fig. 5 

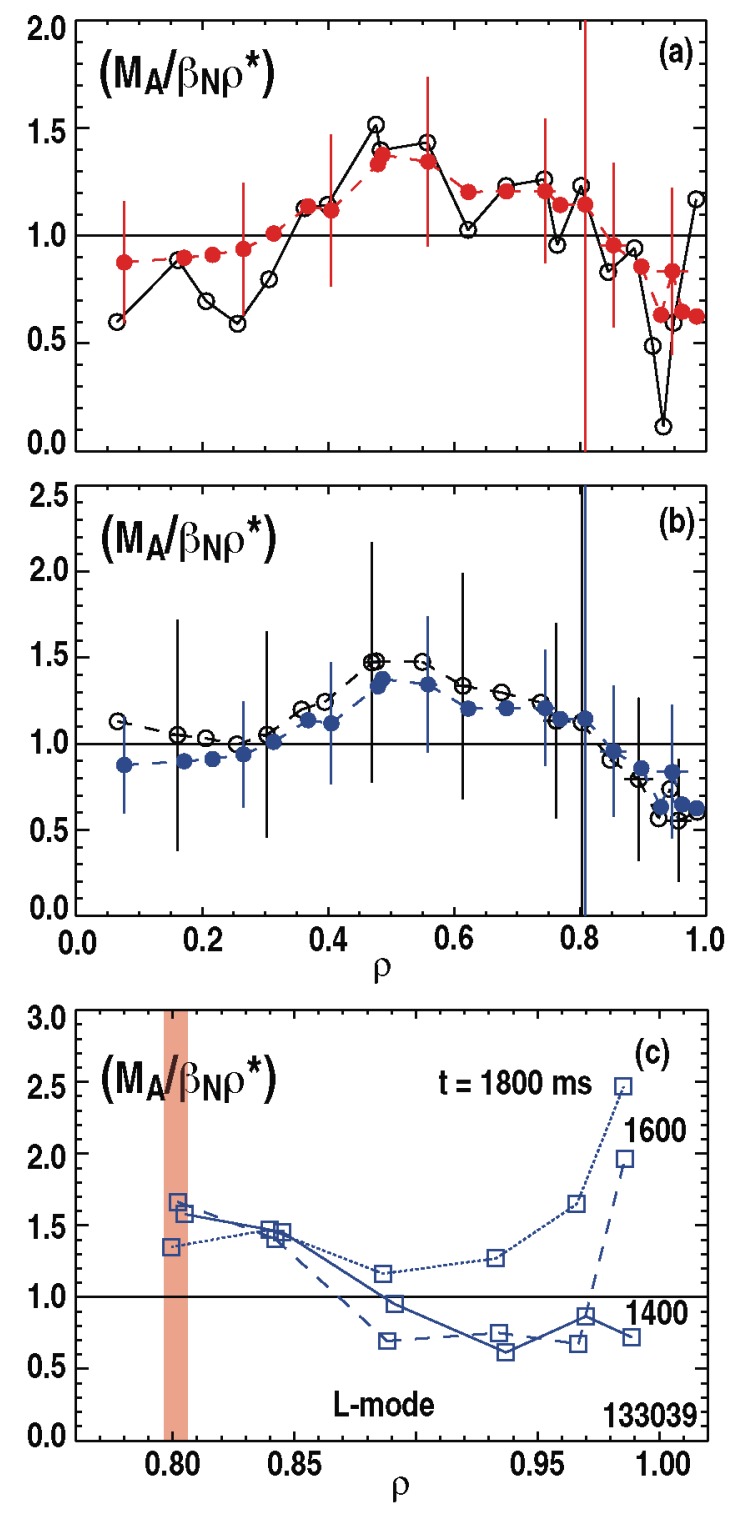

J.S. deGrassie Fig. 6 


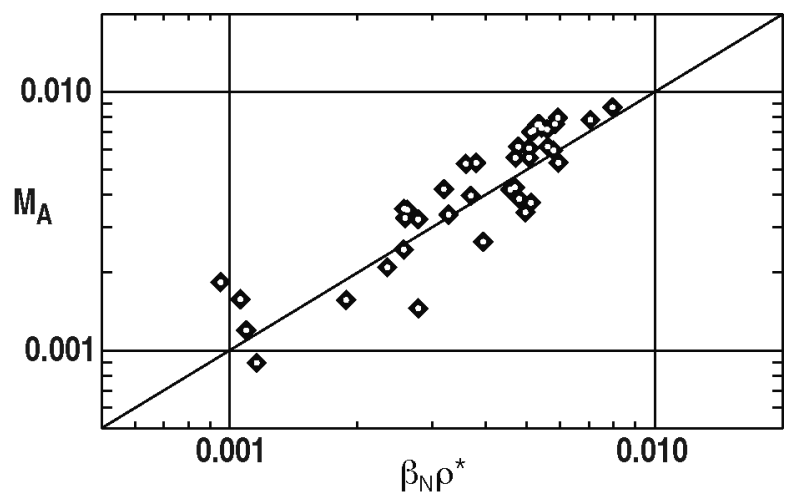

J.S. deGrassie Fig. 7 


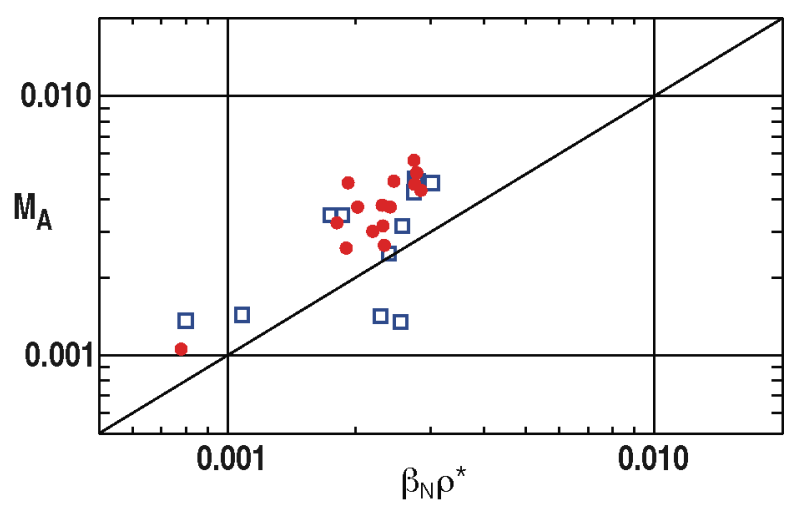

J.S. deGrassie Fig. 8 


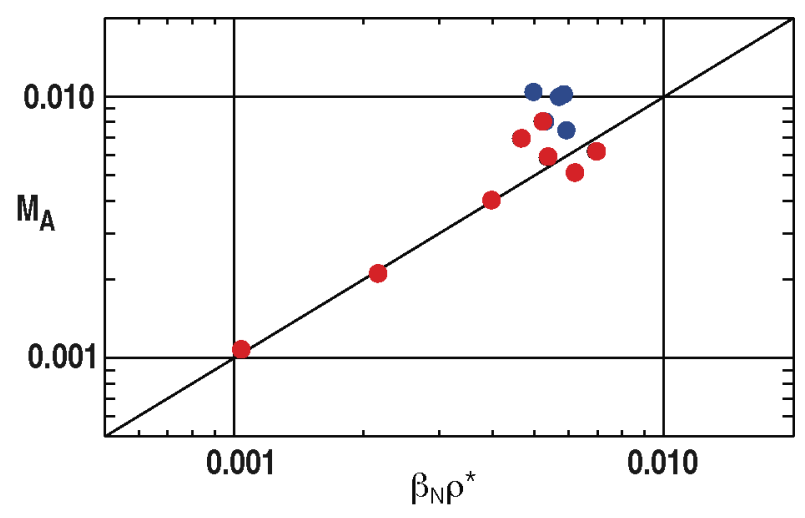

J.S. deGrassie Fig. 9 


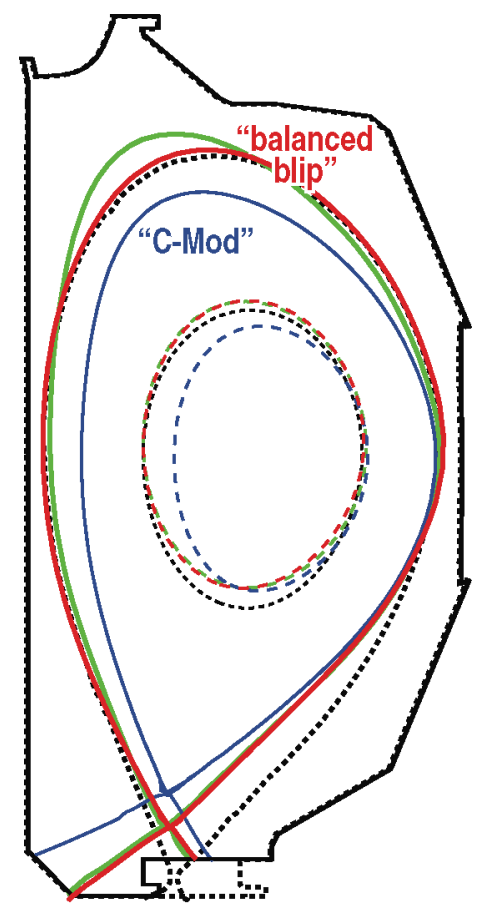

J.S. deGrassie Fig. 10 


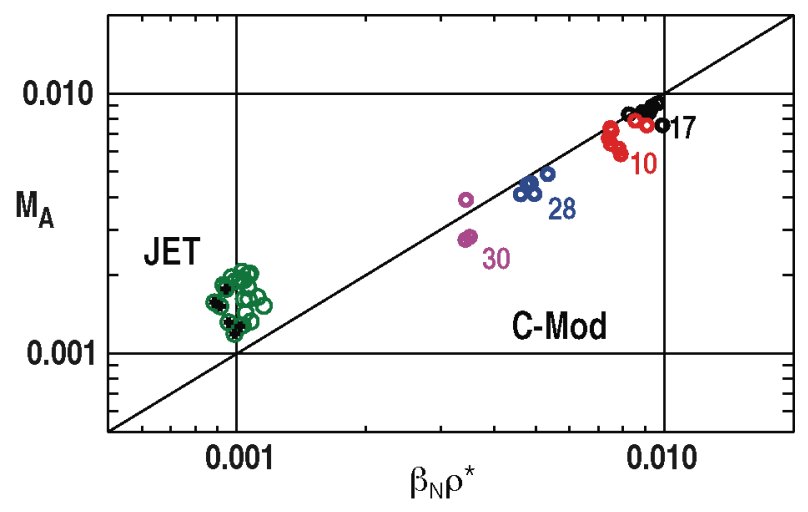

J.S. deGrassie Fig. 11 


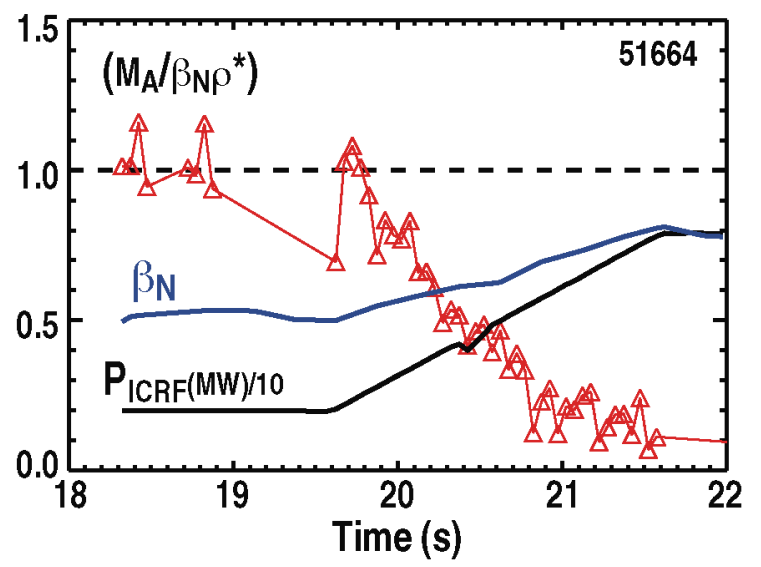

J.S. deGrassie Fig. 12 


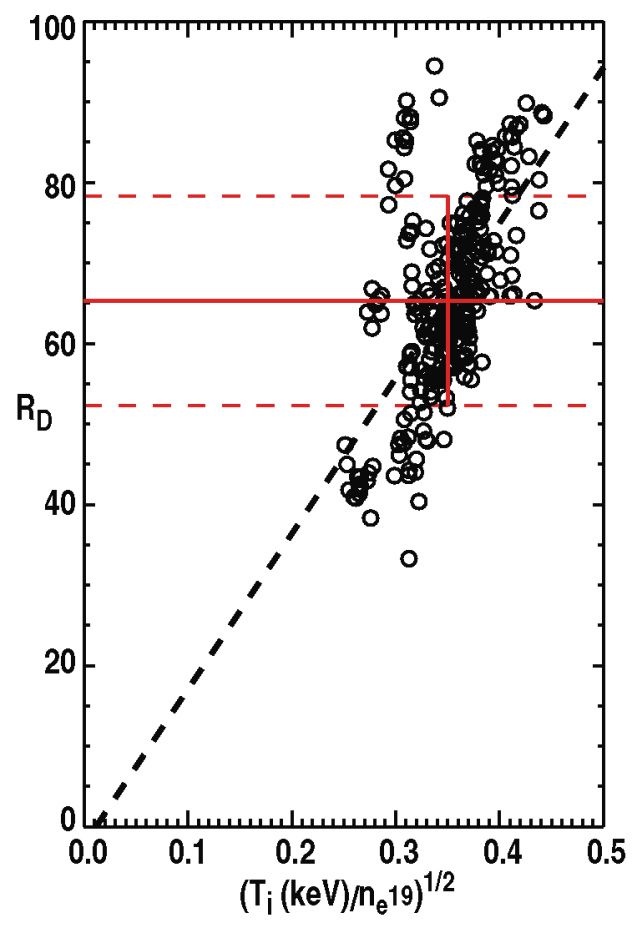

J.S. deGrassie Fig. 13 


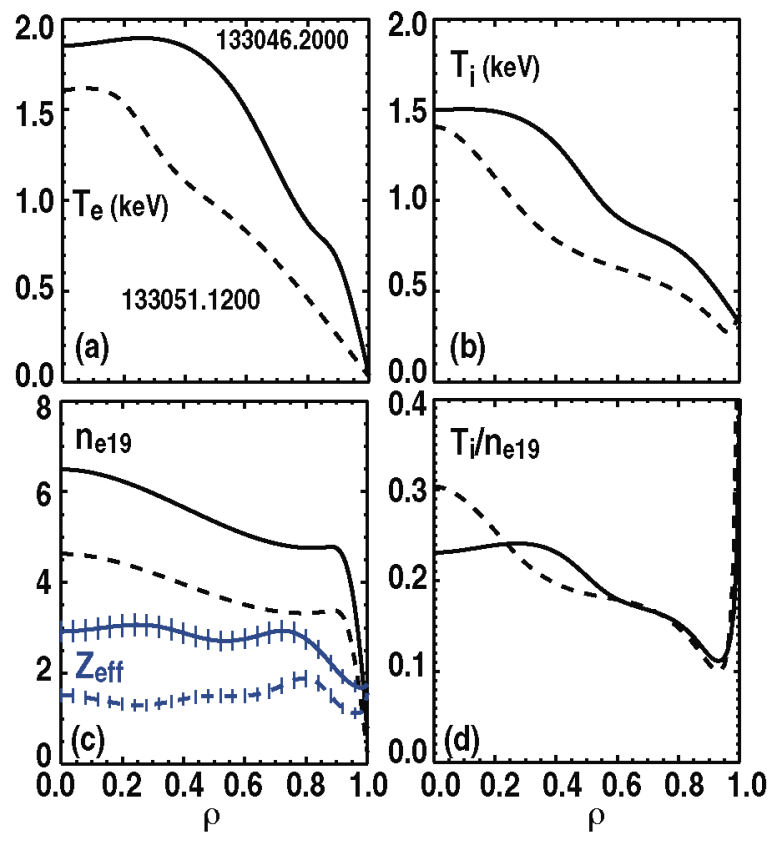

J.S. deGrassie Fig. 14 


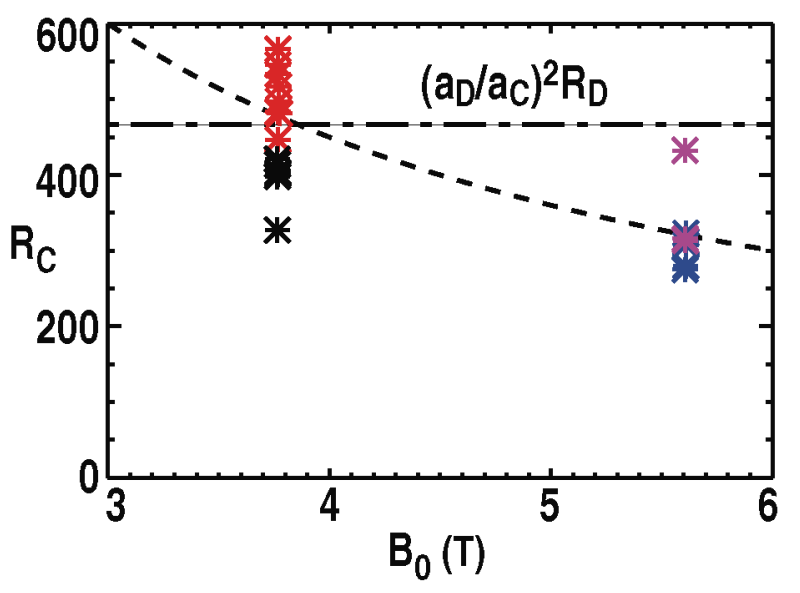

J.S. deGrassie Fig. 15 


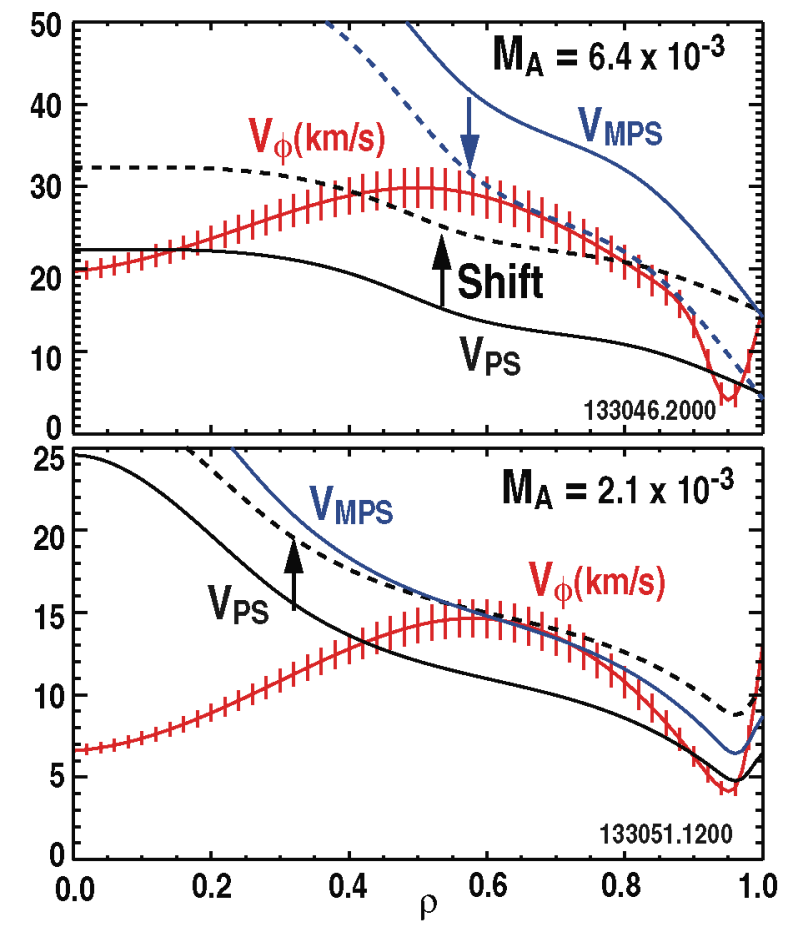

J.S. deGrassie Fig. 16 


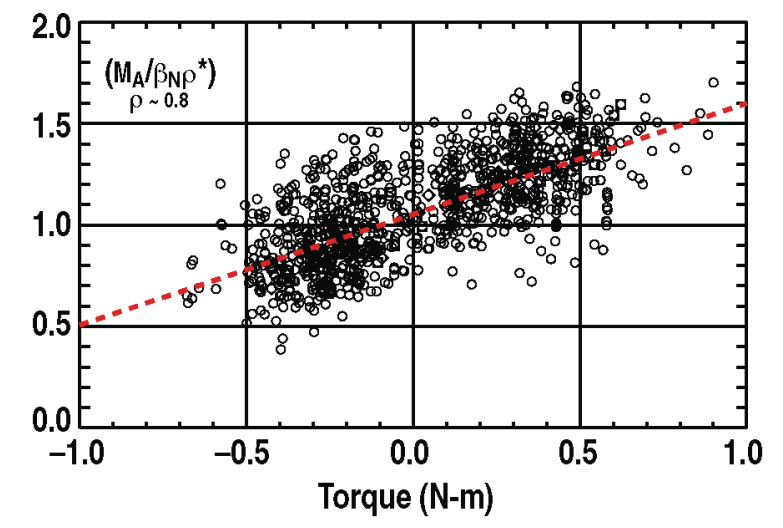

J.S. deGrassie Fig. 17 